This is an electronic reprint of the original article. This reprint may differ from the original in pagination and typographic detail.

Author(s): Hencl, Stanislav; Tengvall, Ville

Title: $\quad$ Sharpness of the differentiability almost everywhere and capacitary estimates for Sobolev mappings

Year: $\quad 2017$

Version:

Please cite the original version:

Hencl, S. \& Tengvall, V. (2017) Sharpness of the differentiability almost everywhere and capacitary estimates for Sobolev mappings. Revista Matemática Iberoamericana, 33 (2), 595-622. doi:10.4171/RMI/951

All material supplied via JYX is protected by copyright and other intellectual property rights, and duplication or sale of all or part of any of the repository collections is not permitted, except that material may be duplicated by you for your research use or educational purposes in electronic or print form. You must obtain permission for any other use. Electronic or print copies may not be offered, whether for sale or otherwise to anyone who is not an authorised user. 


\title{
SHARPNESS OF THE DIFFERENTIABILITY ALMOST EVERYWHERE AND CAPACITARY ESTIMATES FOR SOBOLEV MAPPINGS
}

\author{
STANISLAV HENCL AND VILLE TENGVALL
}

\begin{abstract}
We give sharp conformal conditions for the differentiability in the Sobolev space $W_{\text {loc }}^{1, n-1}\left(\Omega, \mathbb{R}^{n}\right)$. Furthermore, we show that the space $W_{\text {loc }}^{1, n-1}\left(\Omega, \mathbb{R}^{n}\right)$ can be considered as the borderline space for some capacitary inequalities.
\end{abstract}

\section{INTRODUCTION}

Let $\Omega \subset \mathbb{R}^{n}, n \geq 2$, be a domain and suppose that $f \in W_{\text {loc }}^{1, n-1}\left(\Omega, \mathbb{R}^{n}\right)$ is a homeomorphism. If $n=2$, then the theorem of Gehring and Lehto [2] implies that $f$ is differentiable almost everywhere. Moreover, if we proceed to the case $n \geq 3$ then the Sobolev embedding theorem on spheres implies that every homeomorphism in $W_{\text {loc }}^{1, p}\left(\Omega, \mathbb{R}^{n}\right), p>n-1$, is differentiable almost everywhere, see [13, Theorem 1.2]. On the other hand, Sobolev embedding theorem on spheres does not apply when $f \in W_{\text {loc }}^{1, n-1}\left(\Omega, \mathbb{R}^{n}\right), n \geq 3$, which may cause the failure of the differentiability in a set of positive measure. Indeed, in [1, Example 5.2] Csörnyei, Hencl and Malý constructed a nowhere differentiable homeomorphism $f \in W_{\text {loc }}^{1, n-1}\left((-1,1)^{n}, \mathbb{R}^{n}\right), n \geq 3$, of finite distortion with nowhere differentiable inverse.

Differentiability of mappings in the Sobolev space $W_{\text {loc }}^{1, n-1}\left(\Omega, \mathbb{R}^{n}\right), n \geq 3$, can be recovered by requiring some integrability of the distortion functions (see Preliminaries for the definition of mappings of finite distortion). Indeed, it follows from [11, Theorem 1.1] together with [12, Lemma 2.1] that every homeomorphism in $W_{\text {loc }}^{1, n-1}\left(\Omega, \mathbb{R}^{n}\right)$ with locally integrable inner distortion function is differentiable almost everywhere. The topological assumptions above can be further relaxed by assuming $f$ to be only continuous, discrete (the set $f^{-1}(y)$ is a discrete set in $\Omega$ for every $y \in \mathbb{R}^{n}$ ) and open $\left(f(A)\right.$ is an open set in $\mathbb{R}^{n}$ for every open set $A$ in $\left.\Omega\right)$, see [17]. It was asked in [17] whether the local integrability assumption of the inner distortion function above is sharp. We will now give a positive answer to this question by a novel construction:

Theorem 1.1. Let $0<\delta<1$ and $n \geq 3$. Then there is a homeomorphism $f \in W^{1, n-1}\left((-1,1)^{n}, \mathbb{R}^{n}\right)$ with $K_{I} \in L^{\delta}\left((-1,1)^{n}\right)$ such that $f$ is not classically differentiable on a set of positive measure.

When we are studying analytical properties, such as differentiability almost everywhere and continuity, of mappings of finite distortion we are commonly lead to study the geometry of these mappings via weighted capacitary inequalities. One of the

1991 Mathematics Subject Classification. 26B05, 30C65, 46E35.

Both authors were supported by the ERC CZ grant LL1203 of the Czech Ministry of Education. The second author was also supported by the Vilho, Yrjö and Kalle Väisälä foundation. 
most well-known weighted capacitary inequalities in the study of mappings of finite distortion is the $K_{I}$-inequality, also known as the Poletsky-type capacity inequality:

Definition 1.2. Let $f \in W_{\text {loc }}^{1,1}\left(\Omega, \mathbb{R}^{n}\right)$ be a continuous and open mapping of finite distortion with locally integrable inner distortion function. We say that $f$ satisfies the $K_{I}$-inequality if for every condenser $(G, E)$ in $\Omega$ we have

$$
\operatorname{cap}(f(G), f(E)) \leq C_{I}(G) \operatorname{cap}_{K_{I}}(G, E),
$$

where the constant $C_{I} \geq 0$ depends only on the dimension $n$ and on the maximum multiplicity $N(f, G):=\sup _{y \in \mathbb{R}^{n}}$ card $f^{-1}(y) \cap G$. For the terminology used above we refer the reader to the Preliminaries.

We remark that inequality (1.1) is enough to guarantee differentiability almost everywhere for a continuous, discrete and open mapping, and especially for a homeomorphism, of finite distortion, see $[15,16]$. It is also important to notice that the best possible constant $C_{I}(G)$ plays an important role in several applications. Moreover, inequalities similar to (1.1) has been applied widely to study properties of quasiregular mappings, see e.g. $[9,8,10]$.

In the case of quasiregular mappings $K_{I}$-inequality with the constant $C_{I}(G) \equiv 1$ was first proved by Martio, Rickman and Väisälä [10]. Later Martio improved this result by improving the constant $C_{I}(G)$, see [9]. In [7] Koskela and Onninen generalized this inequality for continuous, discrete and open mappings of finite distortion in $W_{\text {loc }}^{1, n}\left(\Omega, \mathbb{R}^{n}\right)$ with a locally integrable inner distortion function. It was further shown that the regularity assumption $f \in W_{\text {loc }}^{1, n}\left(\Omega, \mathbb{R}^{n}\right)$ can be slightly relaxed, say to $|D f|^{n} \log ^{-1}(e+|D f|) \in L_{\text {loc }}^{1}(\Omega)$. These results by Koskela and Onninen were based on a duality argument, relying on integration by parts against the Jacobian determinant. This method does not work if we assume $f \in W_{\text {loc }}^{1, p}\left(\Omega, \mathbb{R}^{n}\right)$ for some $p \in[1, n)$.

In [17, Lemma 4.4] it was shown by applying Ziemer's duality equation [20, 21] that for spherical condensers the $K_{I^{-}}$-inequality is true even for continuous, open and discrete mappings of finite distortion in $W_{\text {loc }}^{1, n-1}\left(\Omega, \mathbb{R}^{n}\right)$ with locally integrable inner distortion function. We will show that the space $W_{\text {loc }}^{1, n-1}\left(\Omega, \mathbb{R}^{n}\right)$ can be considered as the borderline space for the $K_{I}$-inequality:

Theorem 1.3. Let $n \geq 3$ and $\varepsilon>0$. Then there is a homeomorphism $f \in$ $W^{1, n-1-\varepsilon}\left((-1,1)^{n}, \mathbb{R}^{n}\right)$ with $K_{I} \in L^{1}\left((-1,1)^{n}\right)$ for which the $K_{I}$-inequality (1.1) fails.

The main idea for the proof of Theorem 1.3 is to construct a homeomorphism in $W^{1, n-1-\varepsilon}\left((-1,1)^{n}, \mathbb{R}^{n}\right)$ with the given conformality conditions which does not satisfy the Lusin's condition $(N)$ on almost every hyperplane with respect to $(n-1)$ dimensional Hausdorff measure. In our construction we will actually destroy the Lusin's condition on every hyperplane. The reason for this comes from the proof of [17, Lemma 4.4] where the Sobolev regularity of a mapping was only used to show that mapping satisfy the condition $(N)$ on almost every hyperplane.

In the proof of Theorem 1.3 we will apply probability-based techniques to calculate $L^{p}$-norms of distortion functions and differential matrices. More precisely, we will apply the notion of expected value and the famous Khintchine inequality [6] to calculate integrals in Theorem 1.3. As far as we know this is the first time that Khintchine inequality is used to study mappings of finite distortion. 


\section{PRELIMINARIES}

2.1. Notation. We will denote by $C:=C\left(p_{1}, \ldots, p_{k}\right)$ a positive constant which depends only on the given parameters $p_{1}, \ldots p_{k}$. The constant $C$ might change from line to line. Furthermore, for given functions $f$ and $g$ we denote $f \lesssim g$ if there exists a positive constant $C>0$ such that $f(x) \leq C g(x)$ for all points $x$. If both conditions $f \lesssim g$ and $g \lesssim f$ are satisfied we denote $f \sim g$.

2.2. Mappings of finite distortion. Let $\Omega \subset \mathbb{R}^{n}, n \geq 2$ be a domain. We recall that a mapping $f \in W_{\text {loc }}^{1,1}\left(\Omega, \mathbb{R}^{n}\right)$ is having finite distortion if

(1) $J_{f} \in L_{\text {loc }}^{1}(\Omega)$,

(2) $J_{f}(x) \geq 0$ for almost every $x \in \Omega$, and

(3) $D f(x)$ vanishes almost everywhere in the zero set of $J_{f}(x)=\operatorname{det} D f(x)$.

With such a mapping $f$ we may associate the distortion function $K_{I}: \Omega \rightarrow[1, \infty]$ as follows:

$$
K_{I}(x)= \begin{cases}\frac{\left|D^{\sharp} f(x)\right|^{n}}{J_{f}(x)^{n-1}}, & \text { if } J_{f}(x)>0 \\ 1, & \text { otherwise. }\end{cases}
$$

We call the function $K_{I}$ as the inner distortion functions of $f$. Above, $D^{\sharp} f$ stands for the adjugate matrix of the differential matrix $D f$ and $|A|$ stands for the operator norm of a matrix $A$. When $K_{I} \in L^{\infty}(\Omega)$ we call mapping $f$ quasiregular.

2.3. Condensers and the capacity. Suppose that $\Omega \subset \mathbb{R}^{n}, n \geq 2$, is a domain. A pair $(G, E)$ of sets is called as a condenser in $\Omega$ if

(1) $G \subset \subset \Omega$ is a domain, and

(2) $E \subset G$ is a non-empty, compact subset of $G$.

Condenser $(G, E)$ is called spherical if both $G$ and $E$ are balls centered at the same point.

We define the $\omega$-weighted capacity of a condenser $(G, E)$ for a non-negative weight function $\omega \in L^{1}(G)$ as

$$
\operatorname{cap}_{\omega}(G, E):=\inf \left\{\int_{G}|\nabla u(x)|^{n} \omega(x) \mathrm{d} x: u \in C_{0}^{\infty}(G), u \geq 0 \text { and } u \geq 1 \text { on } E\right\} .
$$

In the case $\omega \equiv 1$ we write $\operatorname{cap}(G, E)$ instead of $\operatorname{cap}_{1}(G, E)$.

2.4. Algorithm for constructing Cantor sets. Suppose that $[-1,1]^{m} \subset \mathbb{R}^{m}$, and denote by $\mathbb{V}$ the set of $2^{m}$ vertices of the cube $[-1,1]^{m}$. The sets

$$
\mathbb{V}^{k}=\mathbb{V} \times \cdots \times \mathbb{V}, \quad k \in \mathbb{N}
$$

will serve as the set of indices for our construction.

Next, suppose that $\left\{a_{k}\right\}_{k=0}^{\infty}$ is a decreasing sequence such that $1=a_{0} \geq a_{1} \geq \cdots>$ 0 , and define

$$
r_{k}=2^{-k} a_{k} .
$$

Set $z_{0}=0$. Then it follows that $Q\left(z_{0}, r_{0}\right)=(-1,1)^{m}$ and further we proceed by induction. For $\boldsymbol{v}(k)=\left[v_{1}, \ldots, v_{k}\right] \in \mathbb{V}^{k}$ we denote $\boldsymbol{w}(k)=\left[v_{1}, \ldots, v_{k-1}\right]$ and we 
define

$$
\begin{aligned}
& z_{\boldsymbol{v}(k)}=z_{\boldsymbol{w}(k)}+\frac{1}{2} r_{k-1} v_{k}=z_{0}+\frac{1}{2} \sum_{j=1}^{k} r_{j-1} v_{j}, \\
& Q_{\boldsymbol{v}(k)}^{\prime}=Q\left(z_{\boldsymbol{v}(k)}, 2^{-k} a_{k-1}\right) \text { and } Q_{\boldsymbol{v}(k)}=Q\left(z_{\boldsymbol{v}(k)}, 2^{-k} a_{k}\right) .
\end{aligned}
$$

Then for the measure of the $k$ th frame $Q_{\boldsymbol{v}(k)}^{\prime} \backslash Q_{\boldsymbol{v}(k)}$ we have

$$
\mathcal{L}^{m}\left(Q_{\boldsymbol{v}(k)}^{\prime} \backslash Q_{\boldsymbol{v}(k)}\right)=2^{-k m}\left(a_{k-1}^{m}-a_{k}^{m}\right) .
$$

Formally we should write $\boldsymbol{w}(\boldsymbol{v}(k))$ instead of $\boldsymbol{w}(k)$ but for the simplification of the notation we will avoid this.
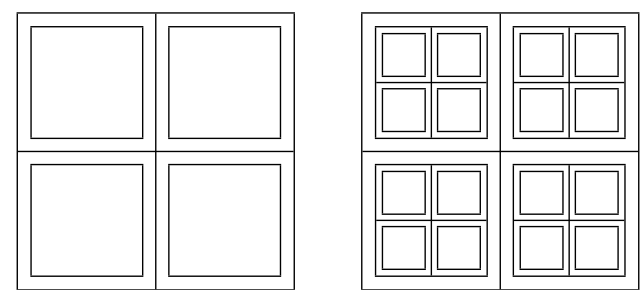

Fig. 1. Cubes $Q_{\boldsymbol{v}(k)}$ and $Q_{\boldsymbol{v}(k)}^{\prime}$ for $k=1,2$.

It is not difficult to find out that the resulting Cantor set

$$
\bigcap_{k=1}^{\infty} \bigcup_{\boldsymbol{v}(k) \in \mathbb{V}^{k}} Q_{\boldsymbol{v}(k)}=: C\left[\left\{a_{k}\right\}_{k=0}^{\infty}\right]=\mathcal{C}_{a} \times \cdots \times \mathcal{C}_{a}
$$

is a product of $m$ Cantor sets $\mathcal{C}_{a}$ in $\mathbb{R}$, and the number of the cubes $\left\{Q_{\boldsymbol{v}(k)}: \boldsymbol{v}(k) \in\right.$ $\left.\mathbb{V}^{k}\right\}$ is $2^{m k}$. Therefore, the measure of the Cantor set $C_{A}:=C\left[\left\{a_{k}\right\}_{k}\right]$ can be calculated as

$$
\mathcal{L}^{m}\left(C_{A}\right)=\lim _{k \rightarrow \infty} 2^{m k}\left(2 a_{k} 2^{-k}\right)^{m}=\lim _{k \rightarrow \infty} 2^{m} a_{k}^{m} .
$$

2.5. Canonical parametrizations. To prove Theorem 1.3 we need to find a way to map Cantor set onto another Cantor set by a homeomorphism. We will do this by using so-called canonical parametrizations and canonical transformations which were first introduced by Hencl, Koskela and Malý in [5].

For a given $z \in \mathbb{R}^{m}$ and $r>0$ we denote

$$
Q_{r}(z):=\left[z_{1}-r, z_{1}+r\right] \times \cdots \times\left[z_{m}-r, z_{m}+r\right] .
$$

We define an affine map $\varphi_{Q_{r}(z)}: Q_{1}(0) \rightarrow Q_{r}(z)$ as

$$
\varphi_{Q_{r}(z)}(x)=r x+z .
$$

Mapping $\varphi_{Q_{r}(z)}$ is called the canonical parametrization of the cube $Q_{r}(z)$.

Furthermore, if $\mathbb{A}=Q_{r^{\prime}}(z) \backslash Q_{r}(z)$ is a cubical annuli, we define

$$
\varphi_{\mathbb{A}}(t, x)=(1-t) \varphi_{Q_{r}(z)}(x)+t \varphi_{Q_{r^{\prime}}(z)}(x), \quad(t, x) \in[0,1] \times \partial Q_{1}(0) .
$$

Mapping $\varphi_{\mathbb{A}}$ is called as the canonical parametrization of a cubical annuli $\mathbb{A}$. 
To calculate $D \varphi_{\mathbb{A}}$ we may distinguish $2^{m}$ different cases depending on which side of the annuli we are. However, by symmetry it suffices to deal with the case $(t, x) \in$ $\left\{(t, x) \in[0,1] \times \partial Q_{1}(0): x_{1}=1\right\}$. In this case, if we denote $d:=(1-t) r+t r^{\prime}$, then we may write

$$
D \varphi_{\mathbb{A}}(t, x)=\left(\begin{array}{cccccc}
r^{\prime}-r & 0 & 0 & \cdots & 0 & 0 \\
\left(r^{\prime}-r\right) x_{2} & d & 0 & \cdots & \vdots & \vdots \\
\left(r^{\prime}-r\right) x_{3} & 0 & d & \cdots & 0 & 0 \\
\vdots & \vdots & \vdots & \ddots & \vdots & \vdots \\
\left(r^{\prime}-r\right) x_{m-1} & 0 & 0 & \cdots & d & 0 \\
\left(r^{\prime}-r\right) x_{m} & 0 & 0 & \cdots & 0 & d
\end{array}\right)
$$

and

$$
\left(D \varphi_{\mathbb{A}}(t, x)\right)^{-1}=\left(\begin{array}{cccccc}
\frac{1}{r^{\prime}-r} & 0 & 0 & \cdots & 0 & 0 \\
-\frac{x_{2}}{d} & \frac{1}{d} & 0 & \cdots & \vdots & \vdots \\
-\frac{x_{3}}{d} & 0 & \frac{1}{d} & \cdots & 0 & 0 \\
\vdots & \vdots & \vdots & \ddots & \vdots & \vdots \\
-\frac{x_{m-1}}{d} & 0 & 0 & \cdots & \frac{1}{d} & 0 \\
-\frac{x_{m}}{d} & 0 & 0 & \cdots & 0 & \frac{1}{d}
\end{array}\right)
$$

2.6. Canonical transformation. Suppose that

$$
\mathbb{A}:=Q_{r^{\prime}}(z) \backslash \operatorname{int}\left(Q_{r}(z)\right) \quad \text { and } \quad \mathbb{B}:=Q_{\tilde{r}^{\prime}}(\tilde{z}) \backslash \operatorname{int}\left(Q_{\tilde{r}^{\prime}}(\tilde{z})\right)
$$

are two cubical annuli. We define the canonical transformation of $\mathbb{A}$ onto $\mathbb{B}$ as

$$
\varphi_{\mathbb{A}, \mathbb{B}}=\varphi_{\mathbb{B}} \circ \varphi_{\mathbb{A}}^{-1} .
$$

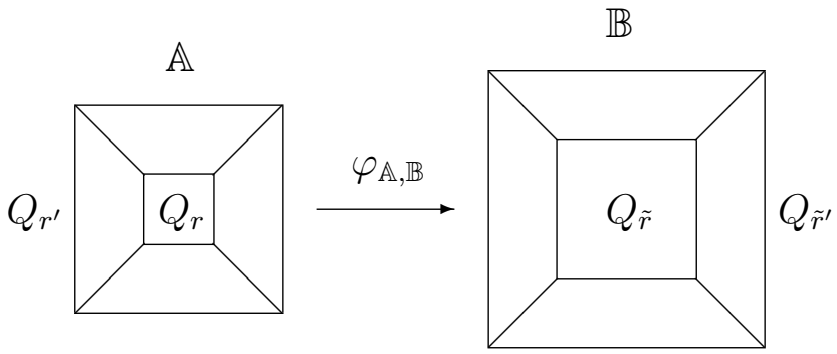

Fig. 2. The canonical transformation of $\mathbb{A}$ onto $\mathbb{B}$ for $m=2$.

Then

$$
D \varphi_{\mathbb{A}, \mathbb{B}}\left(\varphi_{\mathbb{A}}(t, x)\right)=D \varphi_{\mathbb{B}}(x, t)\left(D \varphi_{\mathbb{A}}(x, t)\right)^{-1} .
$$

As in the Section 2.5 when we are calculating the matrix $D \varphi_{\mathbb{A}, \mathbb{B}}\left(\varphi_{\mathbb{A}}(t, x)\right)$ we may distinguish $2^{m}$ different cases depending on which side of the cubical annuli $\mathbb{A}$ we are. However, because of the symmetry we will again write the matrix formally only in the first case of these $2^{m}$ different cases. Then we have 


$$
D \varphi_{\mathbb{A}, \mathbb{B}}\left(\varphi_{\mathbb{A}}(t, x)\right)=\left(\begin{array}{cccccc}
\frac{\tilde{r}^{\prime}-\tilde{r}}{r^{\prime}-r} & 0 & 0 & \cdots & 0 & 0 \\
\left(\frac{\tilde{r}^{\prime}-\tilde{r}}{r^{\prime}-r}-\frac{\tilde{d}}{d}\right)\left(x_{2}-z_{2}\right) & \frac{\tilde{d}}{d} & 0 & \cdots & \vdots & \vdots \\
\left(\frac{\tilde{r}^{\prime}-\tilde{r}}{r^{\prime}-r}-\frac{\tilde{d}}{d}\right)\left(x_{3}-z_{3}\right) & 0 & \frac{\tilde{d}}{d} & \cdots & 0 & 0 \\
\vdots & \vdots & \vdots & \ddots & \vdots & \vdots \\
\left(\frac{\tilde{r}^{\prime}-\tilde{r}}{r^{\prime}-r}-\frac{\tilde{d}}{d}\right)\left(x_{m-1}-z_{m-1}\right) & 0 & 0 & \cdots & \frac{\tilde{d}}{d} & 0 \\
\left(\frac{\tilde{r}^{\prime}-\tilde{r}}{r^{\prime}-r}-\frac{\tilde{d}}{d}\right)\left(x_{m}-z_{m}\right) & 0 & 0 & \cdots & 0 & \frac{\tilde{d}}{d}
\end{array}\right)
$$

where

$$
d=(1-t) r+t r^{\prime} \quad \text { and } \quad \tilde{d}=(1-t) \tilde{r}+t \tilde{r}^{\prime}
$$

2.7. Mapping a Cantor set onto another. Consider two Cantor sets

$$
C_{A}:=C\left[\left\{a_{k}\right\}_{k=0}^{\infty}\right] \quad \text { and } \quad C_{B}:=C\left[\left\{b_{k}\right\}_{k=0}^{\infty}\right]
$$

given by the algorithm introduced in the Section 2.4. Then we may define two sequences of cubical annuli

$$
\mathbb{A}_{\boldsymbol{v}(k)}:=Q_{\boldsymbol{v}(k)}^{\prime} \backslash \operatorname{int}\left(Q_{\boldsymbol{v}(k)}\right) \quad \text { and } \quad \mathbb{B}_{\boldsymbol{v}(k)}:=\tilde{Q}_{\boldsymbol{v}(k)}^{\prime} \backslash \operatorname{int}\left(\tilde{Q}_{\boldsymbol{v}(k)}\right)
$$

where the cubes above are referring to the corresponding ones in the constructions of the Cantor sets $C_{A}$ and $C_{B}$.

We define the homeomorphism $\Phi:(-1,1)^{m} \rightarrow(-1,1)^{m}$ which takes $C_{A}$ onto $C_{B}$ as the pointwise limit of mappings

$$
\Phi_{N}(x)= \begin{cases}\sum_{k=0}^{N} \sum_{\boldsymbol{v}(k) \in \mathbb{V}^{k}} \varphi_{\mathbb{A}_{\boldsymbol{v}(k)}, \mathbb{B}_{\boldsymbol{v}(k)}}(x) \chi_{\mathbb{A}_{\boldsymbol{v}(k)}}(x), & \text { if } x \in \cup_{k=0}^{N} \cup_{\boldsymbol{v}(k) \in \mathbb{V}^{k}} \mathbb{A}_{\boldsymbol{v}(k)} \\ \tilde{\Phi}_{N}(x), & \text { otherwise, }\end{cases}
$$

where $\chi_{E}$ denotes the characteristic function of a set $E$, and $\tilde{\Phi}_{N}$ is a linear mapping which takes each cube $Q_{\boldsymbol{v}(N)}$ linearly onto corresponding cube $\tilde{Q}_{\boldsymbol{v}(N)}$. To see that $\Phi$ defines a homeomorphism we refer the reader to [4, Section 4.3].

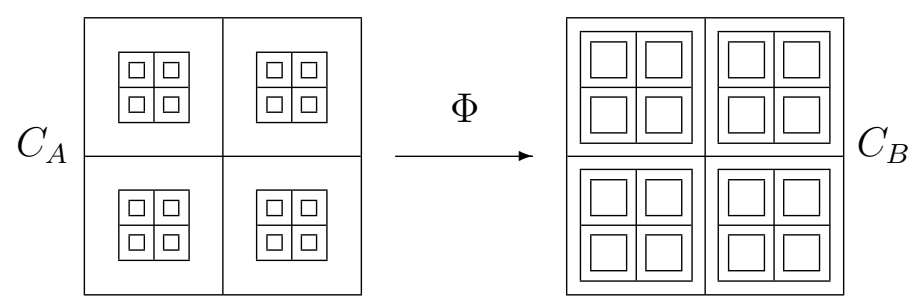

Fig. 3. Mapping the Cantor set $C_{A}$ onto $C_{B}$ by using the mapping $\Phi$.

As in the Sections 2.5 and 2.6 we may again distinguish $2^{m}$ different case when we are calculating $D \Phi$. Again we calculate the differential matrix only in the first of 
these $2^{m}$ cases. In this case we have

$$
D \Phi\left(\varphi_{\mathbb{A}_{\boldsymbol{v}(k)}}(t, x)\right)=\left(\begin{array}{cccccc}
C & 0 & 0 & \cdots & 0 & 0 \\
(C-D)\left(x_{2}-z_{2}\right) & D & 0 & \cdots & \vdots & \vdots \\
(C-D)\left(x_{3}-z_{3}\right) & 0 & D & \cdots & 0 & 0 \\
\vdots & \vdots & \vdots & \ddots & \vdots & \vdots \\
(C-D)\left(x_{m-1}-z_{m-1}\right) & 0 & 0 & \cdots & D & 0 \\
(C-D)\left(x_{m}-z_{m}\right) & 0 & 0 & \cdots & 0 & D
\end{array}\right),
$$

where $z=\left(z_{1}, \ldots, z_{m}\right)$ is the center of $\mathbb{A}_{\boldsymbol{v}(k)}$,

$$
C=\frac{b_{k-1}-b_{k}}{a_{k-1}-a_{k}} \quad \text { and } \quad D=\frac{(1-t) b_{k}+t b_{k-1}}{(1-t) a_{k}+t a_{k-1}} .
$$

2.8. Khintchine-type inequality. The Rademacher's distribution is a discrete probability distribution such that

$$
\mathbb{P}(X=x)= \begin{cases}1 / 2, & \text { if } x=1 \\ 1 / 2, & \text { if } x=-1 \\ 0, & \text { otherwise }\end{cases}
$$

Furthermore, for a given $0 \leq 1 \leq 1$ we define Bernoulli $q$-distribution to be the discrete probability distribution define by

$$
\mathbb{P}(X=x)= \begin{cases}q, & \text { if } x=1 \\ 1-q, & \text { if } x=0 \\ 0, & \text { otherwise. }\end{cases}
$$

Next, assume that $X_{1}$ and $X_{2}$ are two independent random variables, $X_{1}$ has from the Rademacher's distribution and $X_{2}$ has the Bernoulli $q$-distribution. If we define $Y:=X_{1} X_{2}$, then the probability distribution of $Y$ can be written as

$$
\mathbb{P}(Y=y)= \begin{cases}\frac{q}{2}, & \text { if } y=1 \\ \frac{q}{2}, & \text { if } y=-1 \\ 1-q, & \text { if } y=0 \\ 0, & \text { otherwise. }\end{cases}
$$

From now on we will call this distribution as $R B(q)$-distribution. Then we can give a generalized version of the famous Khintchine's inequality [6] in the $R B(q)$-setting. In the case $q=1$ this result will be the usual Khintchine's inequality - see e.g. [6].

Lemma 2.1 (Khinchine-type inequality). Let $0<q \leq 1$. Suppose that $\left\{Y_{k}\right\}_{k=1}^{\infty}$ is a sequence of independent and identically distributed random variables from the $R B(q)$ distribution, and let $\left\{d_{k}\right\}_{k=1}^{\infty}$ be a sequence of real numbers. Then for a given $p>0$ we can estimate the expected value as

$$
\hat{B}_{p, q}\left(\sum_{k} d_{k}^{2}\right)^{1 / 2} \leq\left(\mathbb{E}\left[\left|\sum_{k} d_{k} Y_{k}\right|^{p}\right]\right)^{\frac{1}{p}} \leq \hat{A}_{p, q}\left(\sum_{k} d_{k}^{2}\right)^{1 / 2},
$$

where the constants $\hat{A}_{p, q}$ and $\hat{B}_{p, q}$ are depending only on $p$ and $q$.

Proof. For the convenience of the reader we include the proof of this estimate in the case $p>1$ which follows directly the approach of [19, Proposition 4.5]. There will be three steps in the proof: 
(i) Let us first assume that $p=2$. Recall, that we may write

$$
Y_{k}=X_{k, 1} X_{k, 2}, \quad k \in \mathbb{N},
$$

where $X_{k, 1}$ are random variables from the Rademacher's distribution, $X_{k, 2}$ are random variables from the Bernoulli $q$-distribution, and all the random variables $X_{k, j}$ are independent. By using this fact and independence we get

$$
\begin{aligned}
\mathbb{E}\left[\left|\sum_{k} d_{k} Y_{k}\right|^{2}\right] & =\sum_{k} d_{k}^{2} \mathbb{E}\left[Y_{k}^{2}\right]+2 \sum_{k \neq j} d_{k} d_{j} \mathbb{E}\left[Y_{k}\right] \mathbb{E}\left[Y_{j}\right]=\sum_{k} d_{k}^{2} \mathbb{E}\left[Y_{k}^{2}\right] \\
& =\sum_{k} d_{k}^{2} \mathbb{E}\left[X_{k, 1}^{2}\right] \mathbb{E}\left[X_{k, 2}^{2}\right]=q \sum_{k} d_{k}^{2} .
\end{aligned}
$$

(ii) For the upper bound we notice that for every $t>0$ we may estimate

$$
\mathbb{E}\left[e^{t \sum_{k} d_{k} Y_{k}}\right]=\prod_{k} \mathbb{E}\left[e^{t d_{k} Y_{k}}\right] \leq 2 \prod_{k=1}^{N} \frac{1}{2}\left(e^{t d_{k}}+e^{-t d_{k}}\right),
$$

and by applying the numerical inequality $\frac{1}{2}\left(e^{x}+e^{-x}\right) \leq e^{\frac{x^{2}}{2}}$ we conclude

$$
\mathbb{E}\left[e^{t \sum_{k} d_{k} Y_{k}}\right] \leq 2 e^{\frac{t^{2}}{2} \sum_{k} d_{k}^{2}} .
$$

By applying Chebychev's inequality to this estimate, we get

$$
\mathbb{P}\left(\sum_{k} d_{k} Y_{k} \geq \lambda\right) \leq e^{-t \lambda} \mathbb{E}\left[e^{t \sum_{k} d_{k} Y_{k}}\right] \leq 2 e^{-t \lambda+\frac{t^{2}}{2} \sum_{k} d_{k}^{2}}
$$

for any $t>0$ and $\lambda>0$. Taking $t=\frac{\lambda}{\sum_{k} d_{k}^{2}}$ gives

$$
\mathbb{P}\left(\sum_{k} d_{k} Y_{k} \geq \lambda\right) \leq 2 e^{-\frac{\lambda^{2}}{2 \sum_{k} d_{k}^{2}}}
$$

and hence

$$
\mathbb{P}\left(\left|\sum_{k} d_{k} Y_{k}\right| \geq \lambda\right) \leq 4 e^{-\frac{\lambda^{2}}{2 \sum_{k} d_{k}^{2}}}
$$

By applying Cavalieri's principle, we get

$$
\begin{aligned}
\mathbb{E}\left[\left|\sum_{k} d_{k} Y_{k}\right|^{p}\right] & =p \int_{0}^{\infty} \lambda^{p-1} \mathbb{P}\left(\left|\sum_{k} d_{k} Y_{k}\right| \geq \lambda\right) \mathrm{d} \lambda \\
& \leq 4 p \int_{0}^{\infty} \lambda^{p-1} e^{-\frac{\lambda^{2}}{2 \sum_{k} d_{k}^{2}}} \mathrm{~d} \lambda=C\left(\sum_{k} d_{k}^{2}\right)^{\frac{p}{2}}
\end{aligned}
$$

where the constant $C>0$ is depending only on $p$. This gives us the upper bound.

(iii) The lower bound follows from (i), (ii) and Hölder's inequality:

$$
\begin{aligned}
\sum_{k} d_{k}^{2} \sim \mathbb{E}\left[\left|\sum_{k} d_{k} Y_{k}\right|^{2}\right] & \leq\left(\mathbb{E}\left[\left|\sum_{k} d_{k} Y_{k}\right|^{p}\right]\right)^{\frac{1}{p}}\left(\mathbb{E}\left[\left|\sum_{k} d_{k} Y_{k}\right|^{\frac{p}{p-1}}\right]\right)^{\frac{p-1}{p}} \\
& \lesssim\left(\mathbb{E}\left[\left|\sum_{k} d_{k} Y_{k}\right|^{p}\right]\right)^{\frac{1}{p}}\left(\sum_{k} d_{k}^{2}\right)^{\frac{1}{2}}
\end{aligned}
$$


and we have

$$
\left(\sum_{k} d_{k}^{2}\right)^{\frac{1}{2}} \lesssim\left(\mathbb{E}\left[\left|\sum_{k} d_{k} Y_{k}\right|^{p}\right]\right)^{\frac{1}{p}}
$$

2.9. Oscillating snowflake mapping on a Cantor set. Let $C_{A}:=C\left[\left\{a_{k}\right\}_{k=0}^{\infty}\right]$ be a Cantor set in $\mathbb{R}^{m}$. We recall that we may write every index $\boldsymbol{v} \in \mathbb{V}^{k}$ as

$$
\boldsymbol{v}=\left(\boldsymbol{v}_{1}, \boldsymbol{v}_{2}, \ldots, \boldsymbol{v}_{k}\right)=(\underbrace{\boldsymbol{v}_{1,1}, \ldots, \boldsymbol{v}_{1, m}}_{=\boldsymbol{v}_{1} \in \mathbb{V}}, \underbrace{\boldsymbol{v}_{2,1}, \ldots, \boldsymbol{v}_{2, m}}_{=\boldsymbol{v}_{2} \in \mathbb{V}}, \ldots, \underbrace{\boldsymbol{v}_{k, 1}, \ldots, \boldsymbol{v}_{k, m}}_{=\boldsymbol{v}_{m} \in \mathbb{V}}),
$$

where $\boldsymbol{v}_{i, j} \in\{-1,1\}$. Thus, with every $\boldsymbol{v} \in \mathbb{V}^{k}$ we may associate a number $\delta(\boldsymbol{v}) \in$ $\{-1,1\}$ as

$$
\delta(\boldsymbol{v}):=\prod_{j=1}^{m} \boldsymbol{v}_{k, j},
$$

which will play a role of a sign function in our construction.
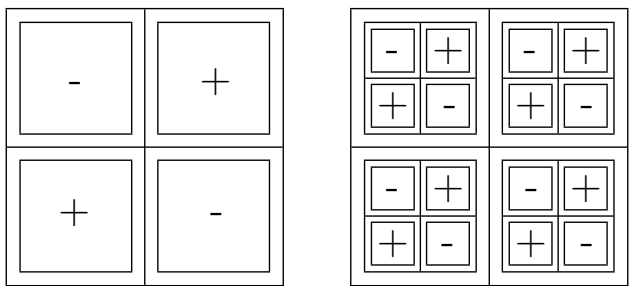

Fig. 4. Sign of $\delta(\boldsymbol{v})$.

Suppose that $\left\{d_{k}\right\}_{k=1}^{\infty}$ is a sequence of real numbers converging to zero. Then we define a sequence $\left\{S_{k}\right\}_{k=1}^{\infty}$ of mappings $S_{k}:(-1,1)^{m+1} \rightarrow \mathbb{R}^{m+1}$ as

$$
S_{k}\left(x_{1}, \ldots, x_{m}, x_{m+1}\right)=\left(x_{1}, \ldots, x_{m}, x_{m+1}+\sum_{\boldsymbol{v} \in \mathbb{V} k} \delta(\boldsymbol{v}) H_{Q \boldsymbol{v}}^{d_{k}}(\hat{x})\right),
$$

where $\hat{x}:=\left(x_{1}, \ldots, x_{m}\right)$ and

$$
H_{Q v}^{d_{k}}(\hat{x})= \begin{cases}d_{k} \inf _{z \in \partial Q_{v}}|\hat{x}-z|, & \text { if } \hat{x} \in Q_{\boldsymbol{v}} \\ 0, & \text { otherwise. }\end{cases}
$$

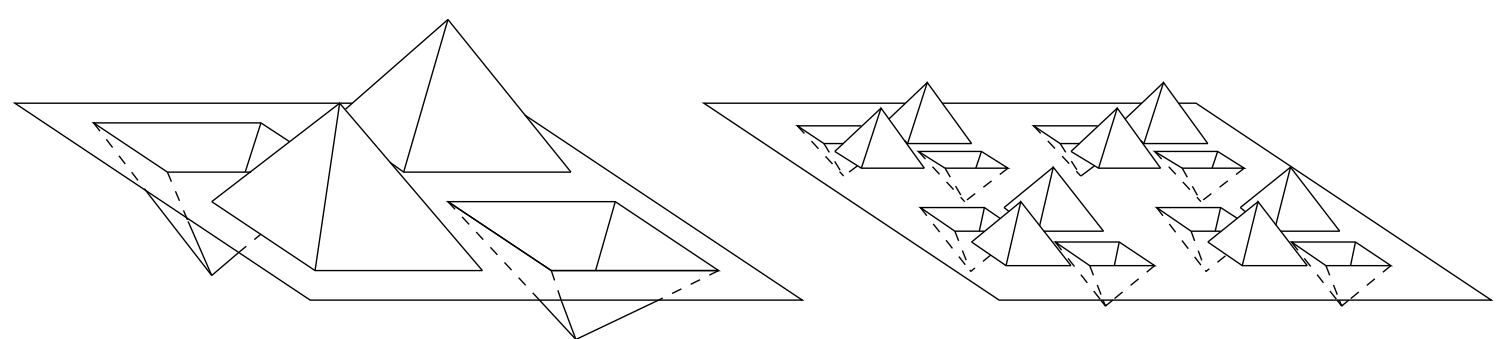

Fig. 5. Image of a 2-dimensional hyperplane for mappings $S_{1}$ and $S_{2}$.

Then we may define mappings $\mathcal{S}_{N}:(-1,1)^{m+1} \rightarrow \mathbb{R}^{m+1}$ as

$$
\mathcal{S}_{N}(x):=\left(S_{N} \circ S_{N-1} \circ \cdots \circ S_{1}\right)(x),
$$


and set $\mathcal{S}(x):=\lim _{N \rightarrow \infty} \mathcal{S}_{N}(x)$. This limit exists for all $x \in(-1,1)^{m+1}$ because

$$
\sum_{k}\left|d_{k} r_{k}\right|=\sum_{k}\left|d_{k} 2^{-k} a_{k}\right|<\infty
$$

Furthermore, it is not hard to see that $\mathcal{S}:(-1,1)^{m+1} \rightarrow \mathcal{S}\left((-1,1)^{m+1}\right)$ is a homeomorphism.

2.10. Properties of $D \mathcal{S}$. To calculate $D \mathcal{S}(x)$ we define sets

$$
\begin{aligned}
& Q_{\boldsymbol{v}}^{i}:=\left\{x \in Q_{\boldsymbol{v}}:\left|\frac{x_{j}-\left(z_{\boldsymbol{v}}\right)_{j}}{x_{i}-\left(z_{\boldsymbol{v}}\right)_{i}}\right|<1 \text { for all } j \neq i\right\}, \\
& \left(Q_{\boldsymbol{v}}^{i}\right)^{-}:=Q_{\boldsymbol{v}}^{i} \cap\left\{x_{i}>\left(z_{\boldsymbol{v}}\right)_{i}\right\}, \\
& \left(Q_{\boldsymbol{v}}^{i}\right)^{+}:=Q_{\boldsymbol{v}}^{i} \cap\left\{x_{i}<\left(z_{\boldsymbol{v}}\right)_{i}\right\},
\end{aligned}
$$

and indicator functions

$$
\delta_{Q_{v}}^{i}(x)= \begin{cases}1, & \text { if } x \in\left(Q_{\boldsymbol{v}}^{i}\right)^{+} \\ -1, & \text { if } x \in\left(Q_{\boldsymbol{v}}^{i}\right)^{-} \\ 0, & \text { otherwise, }\end{cases}
$$

for every $i=1, \ldots, m$.

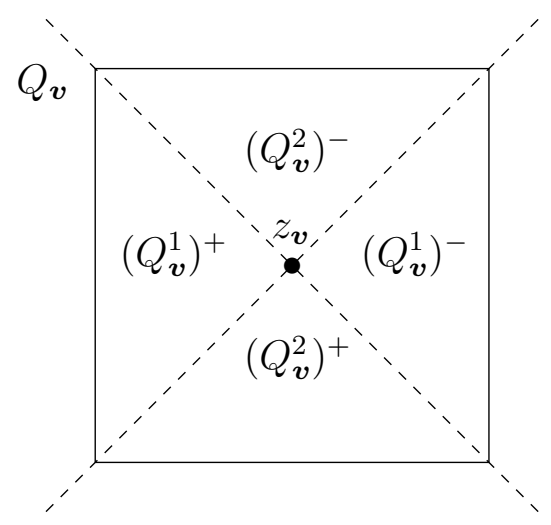

Fig. 6. Sets $\left(Q_{\boldsymbol{v}}^{i}\right)^{-}$and $\left(Q_{\boldsymbol{v}}^{i}\right)^{+}$in the case $m=2$.

For a given point $x=\left(\hat{x}, x_{m+1}\right) \in Q_{\boldsymbol{v}(k)} \times(-1,1)$ the differential matrix $D S_{k}(x)$ can be written as

$$
D S_{k}\left(\hat{x}, x_{m+1}\right)=\left(\begin{array}{ccccc}
1 & 0 & \cdots & 0 & 0 \\
0 & 1 & \cdots & 0 & 0 \\
\vdots & \vdots & \ddots & \vdots & \vdots \\
0 & 0 & \cdots & 1 & 0 \\
d_{k} \delta_{\boldsymbol{v}}^{1} & d_{k} \delta_{\boldsymbol{v}}^{2} & \cdots & d_{k} \delta_{\boldsymbol{v}}^{m} & 1
\end{array}\right)
$$


where $\delta_{\boldsymbol{v}}^{j}:=\delta_{Q_{v}}^{j}(\hat{x}) \delta(\boldsymbol{v})$. Furthermore, by induction we conclude

$$
\begin{aligned}
& D_{k}\left(\hat{x}, x_{m+1}\right)=D S_{k} D \mathcal{S}_{k-1} \\
& =\left(\begin{array}{ccccc}
1 & 0 & \cdots & 0 & 0 \\
0 & 1 & \cdots & 0 & 0 \\
\vdots & \vdots & \ddots & \vdots & \vdots \\
0 & 0 & \cdots & 1 & 0 \\
d_{k} \delta_{\boldsymbol{v}}^{1} & d_{k} \delta_{\boldsymbol{v}}^{2} & \cdots & d_{k} \delta_{\boldsymbol{v}}^{m} & 1
\end{array}\right)\left(\begin{array}{ccccc}
1 & 0 & \cdots & 0 & 0 \\
0 & 1 & \cdots & 0 & 0 \\
\vdots & \vdots & \ddots & \vdots & \vdots \\
0 & 0 & \cdots & 1 & 0 \\
D_{k-1}^{1} & D_{k-1}^{2} & \cdots & D_{k-1}^{m} & 1
\end{array}\right) \\
& =\left(\begin{array}{ccccc}
1 & 0 & \cdots & 0 & 0 \\
0 & 1 & \cdots & 0 & 0 \\
\vdots & \vdots & \ddots & \vdots & \vdots \\
0 & 0 & \cdots & 1 & 0 \\
D_{k}^{1} & D_{k}^{2} & \cdots & D_{k}^{m} & 1
\end{array}\right),
\end{aligned}
$$

where $D_{i}^{j}:=\sum_{l=1}^{i} d_{l} \delta_{Q_{\left(\boldsymbol{v}_{1}, \ldots, \boldsymbol{v}_{l}\right)}}^{j}(\hat{x}) \delta\left(\boldsymbol{v}_{l}\right)$. We also notice that $\delta_{Q_{v}}^{j}(\hat{x})$ and $\delta(\boldsymbol{v})$ are independent random variables, and thus it is easy to see that the probability mass function of $\delta_{\boldsymbol{v}}^{j}$ can be defined as in (2.5) with $q=\frac{1}{n-1}$.

\section{Proof of Theorem 1.1}

In this section we will prove Theorem 1.1. To capture the essential part of the proof we will first give a detailed proof in the case $n=3$ and then sketch the proof in the case $n \geq 4$. Before going to proofs we will describe a basic building block of our construction.

Let $\gamma>0$ be sufficiently small as described later. Let $0<a<\gamma, 0<\eta<\frac{1}{4}$ and $2 a^{\eta}<1 / 2$. Let $h:[0, a] \rightarrow[0,1]$ be defined as

$$
h(t)=\min \left\{\pi-t, \log ^{\frac{1}{4}} \frac{1}{t}-\log ^{\frac{1}{4}} \frac{1}{a}\right\} .
$$

Then $h$ is continuous, strictly decreasing function, piecewise $C^{1}$ with $h(0)=\pi$ and $h(a)=0$. We set

$$
g(t)=-\frac{a^{\eta}}{\pi} h(t)+a^{\eta} .
$$

Now $\left|g^{\prime}(t)\right|=\frac{a^{\eta}}{\pi}\left|h^{\prime}(t)\right|, g(0)=0$ and $g(a)=a^{\eta}$. We define a linear function

$$
\rho(t)=\frac{a^{\eta}}{2 a^{\eta}-a} t+2 a^{\eta} \frac{a^{\eta}-a}{2 a^{\eta}-a} .
$$

Then $\rho\left(2 a^{\eta}\right)=2 a^{\eta}$ and $\rho(a)=a^{\eta}$.

Let us consider three anuloids $T \subset S \subset R$ given by

$$
\begin{aligned}
& T:=\left\{[x, y, z] \in \mathbb{R}^{3}:\left(\sqrt{y^{2}+z^{2}}-1\right)^{2}+x^{2} \leq a^{2}\right\} \\
& S:=\left\{[x, y, z] \in \mathbb{R}^{3}:\left(\sqrt{y^{2}+z^{2}}-1\right)^{2}+x^{2} \leq\left(a^{\eta}\right)^{2}\right\} \text { and } \\
& R:=\left\{[x, y, z] \in \mathbb{R}^{3}:\left(\sqrt{y^{2}+z^{2}}-1\right)^{2}+x^{2} \leq\left(2 a^{\eta}\right)^{2}\right\} .
\end{aligned}
$$

We will define a homeomorphism $F: R \rightarrow R$ such that $F(p)=p$ for every $p \in \partial R$ and $F(\partial T)=\partial S$ is given by the natural stretching. We will use the following system 
of coordinates:

$r$ radius in the anuloid: $r^{2}=\left(\sqrt{y^{2}+z^{2}}-1\right)^{2}+x^{2}$,

$\alpha$ angle in the anuloid: $\sin \alpha=\frac{x}{r}$,

$\beta$ angle around the anuloid: $\sin \beta=\frac{y}{\sqrt{y^{2}+z^{2}}}$,

and we set

$$
\begin{aligned}
& F(r, \alpha, \beta)=[\rho(r), \alpha, \beta] \text { for }[r, \alpha, \beta] \in R \backslash T \text { and } \\
& F(r, \alpha, \beta)=[g(r), \alpha, h(r)+\beta] \text { for }[r, \alpha, \beta] \in T .
\end{aligned}
$$

Lemma 3.1. Let $F: R \rightarrow R$ be as described above in (3.1), and let $0<\delta<1$. Then

(1) $F(p)=p$ for every $p \in \partial R$,

(2) $F$ is a homeomorphism,

(3) $F(\partial T)=\partial S$, and

(4) $|F(p)-p|=2$ for every $p \in\left\{[x, y, z]: y^{2}+z^{2}=1\right.$ and $\left.x=0\right\}$.

Moreover, we have

$$
\begin{gathered}
\int_{R}|D F|^{2} \leq C a^{2 \eta} \log \frac{1}{a}+C \frac{1}{\log ^{\frac{1}{2}} \frac{1}{a}} \text { and } \\
\int_{R} K_{I}^{\delta} \leq C a^{2 \eta} \log \frac{1}{a}+C a^{-\eta \delta+2-2 \delta},
\end{gathered}
$$

where the constant $C>0$ depends only on $n$ and $\delta$.

Proof. We start by verifying the conditions (1)-(4):

(1) By $\rho\left(2 a^{\eta}\right)=2 a^{\eta}$ we obtain $F(p)=p$ for every $p \in \partial R$.

(2) As $\rho(a)=a^{\eta}=g(a)$ and $h(a)=0$ it is easy to check that the mapping is continuous at $\partial T$. By the first line in (3.1), and by applying the facts $\rho\left(2 a^{\eta}\right)=2 a^{\eta}$ and $\rho(a)=a^{\eta}$ we easily see that $F$ is a homeomorphism of $R \backslash T$ onto $R \backslash S$. It is also a homeomorphism of $T$ onto $S$ as $g$ is increasing with $g(0)=0$ and $g(a)=a^{\eta}$ and in the $\beta$ coordinate we have a simple rotation by the angle $h(r)$.

(3) The property $F(\partial T)=\partial S$ easily follows from the arguments in (2).

(4) We note that the middle circle $\left(\left\{y^{2}+z^{2}=1\right.\right.$ and $\left.\left.x=0\right\}\right)$ of $R$ is rotated by the angle of $\pi=h(0)$ and hence we conclude that $|F(p)-p|=2$ there.

We still have to verify (3.2). We express the derivative in the system of coordinates given by $[r, \alpha, \beta]$. The radial direction ( $\alpha$ and $\beta$ fixed and $r$ increases), angular direction inside $(r$ and $\beta$ fixed and $\alpha$ increases) and angular direction around $(r$ and $\alpha$ fixed and $\beta$ increases) are orthogonal and hence we can compute $|D F|$ or $J_{F}$ with respect to these directions. The derivative in the corresponding system of coordinates is given by (see [3, Section 8] for similar computations)

$$
D F(r, \alpha, \beta)=\left(\begin{array}{ccc}
\rho^{\prime}(r) & 0 & 0 \\
0 & \frac{\rho(r)}{r} & 0 \\
0 & 0 & M
\end{array}\right) \quad \text { for }[r, \alpha, \beta] \in R \backslash T
$$


and

$$
D F(r, \alpha, \beta)=\left(\begin{array}{ccc}
g^{\prime}(r) & 0 & h^{\prime}(r) \\
0 & \frac{g(r)}{r} & 0 \\
0 & 0 & M
\end{array}\right) \quad \text { for }[r, \alpha, \beta] \in T,
$$

where $\frac{1-2 a^{\eta}}{1+2 a^{\eta}} \leq|M| \leq \frac{1+2 a^{\eta}}{1-2 a^{\eta}}$ : Let us note that the middle term comes from the fact that the circle of radius $2 \pi r$ is mapped to the circle of radius $2 \pi \rho(r)$ (or $2 \pi g(r)$ in the second case) and hence the derivative around this circle which must be the same as the derivative in the tangential direction to this circle equals $\frac{2 \pi \rho(r)}{2 \pi r}$. The last term with $M$ comes from the fact that the length of any circle around our anuloid (in the $\beta$ direction) has length in $\left[2 \pi\left(1-2 a^{\eta}\right), 2 \pi\left(1+2 a^{\eta}\right)\right]$ and it is mapped to the similar circle around - as we are squeezing in the $r$ direction this is not necessarily the same circle. Note that as $2 a^{\eta}<\frac{1}{2}$ we have $M \sim C$ for some constant $C>0$. Analogously we can check that the normalization of the other terms in the matrix is done correctly.

It is easy to check that on $R \backslash T$ we have $\rho^{\prime}(r) \sim C$ and $\frac{\rho(r)}{r} \geq C$ and hence $|D F| \sim \frac{\rho(r)}{r}$ there.

Using polar coordinates it follows that

$$
\begin{aligned}
\int_{R \backslash T}|D F|^{2} & \leq C \int_{R \backslash T} \frac{\rho^{2}(r)}{r^{2}} \leq C \int_{a}^{2 a^{\eta}} \frac{\rho^{2}(r)}{r^{2}} r \mathrm{~d} r \\
& \leq C a^{2 \eta} \int_{a}^{2 a^{\eta}} \frac{1}{r} \mathrm{~d} r \leq C a^{2 \eta} \log \frac{1}{a} .
\end{aligned}
$$

As $\rho^{\prime}(r) \sim C$ it is easy to see that $\left|D^{\#} F\right| \sim \frac{\rho(r)}{r}$ and $J_{F} \sim \frac{\rho(r)}{r}$ on $R \backslash T$, and hence

$$
\int_{R \backslash T}\left|K_{I}\right|^{\delta} \leq C \int_{R \backslash T}\left(\frac{\rho(r)}{r}\right)^{2 \delta} \leq C \int_{a}^{2 a^{\eta}} \frac{\rho^{2}(r)}{r^{2}} r \mathrm{~d} r \leq C a^{2 \eta} \log \frac{1}{a} .
$$

Now we estimate the derivative on $T$. In the small part around $r=0$ where $h(r)=\pi-r$ we have $\left|g^{\prime}(r)\right|=\frac{a^{\eta}}{\pi}\left|h^{\prime}(r)\right|=\frac{a^{\eta}}{\pi}$ and also $\frac{g(r)}{r}=\frac{a^{\eta}}{\pi}$. It follows that on this set we have $|D F| \leq C$ and

$$
K_{I} \leq \frac{\left(D^{\#} F\right)^{3}}{J_{F}^{2}} \leq C \frac{a^{3 \eta}}{\left(a^{2 \eta}\right)^{2}} \leq C a^{-\eta}
$$

It follows that on this set we have

$$
\int|D F|^{2} \leq C|T| \leq C a^{2} \text { and } \int K_{I}^{\delta} \leq C a^{2} a^{-\delta \eta}
$$

and this causes no trouble for the estimate (3.2).

It remains to estimate the derivative on the main part of $T$ where $h(r)=\log ^{\frac{1}{4}} \frac{1}{r}-$ $\log ^{\frac{1}{4}} \frac{1}{a}$. We can clearly choose $\gamma>0$ small enough at the beginning so that for every $a<\gamma$ we have

$$
\log ^{\frac{1}{4}} \frac{1}{a^{2}}-\log ^{\frac{1}{4}} \frac{1}{a}=\left(\log ^{\frac{1}{4}} \frac{1}{a}\right)(\sqrt[4]{2}-1)>\pi=h(0) .
$$


For $a \leq \gamma$ we thus consider integrals over the set where $r \in\left[a^{2}, a\right]$ as this will only enlarge the set. On this set we have

$$
\left|h^{\prime}(r)\right| \sim \frac{1}{r \log ^{\frac{3}{4}} \frac{1}{r}},\left|g^{\prime}(r)\right|=\frac{a^{\eta}}{\pi}\left|h^{\prime}(r)\right| \sim \frac{a^{\eta}}{r \log ^{\frac{3}{4}} \frac{1}{r}} \text { and } \frac{g(r)}{r} \sim \frac{a^{\eta}}{r} .
$$

It follows that

$$
\int|D F|^{2} \leq C \int_{a^{2}}^{a} \frac{1}{r^{2} \log ^{\frac{3}{2}} \frac{1}{r}} r \mathrm{~d} r+C \int_{a^{2}}^{a} \frac{a^{2 \eta}}{r^{2}} r \mathrm{~d} r \leq C \frac{1}{\log ^{\frac{1}{2}} \frac{1}{a}}+C a^{2 \eta} .
$$

It is not difficult to check that $\left|D^{\#} F\right| \sim\left|h^{\prime}(r)\right| \frac{g(r)}{r}$ and $J_{F} \sim\left|g^{\prime}(r)\right| \frac{g(r)}{r} \sim a^{\eta}\left|h^{\prime}(r)\right| \frac{g(r)}{r}$ on this set and hence

$$
\begin{aligned}
\int K_{I}^{\delta} & \leq C \int_{a^{2}}^{a}\left(\frac{\left|h^{\prime}(r)\right|}{a^{2 \eta}} \frac{g(r)}{r}\right)^{\delta} r \mathrm{~d} r \leq C \int_{a^{2}}^{a}\left(\frac{1}{a^{2 \eta} r \log ^{\frac{3}{4}} \frac{1}{r}} \frac{a^{\eta}}{r}\right)^{\delta} r \mathrm{~d} r \\
& \leq C a^{-\eta \delta} \frac{1}{\log ^{\frac{3}{4} \delta} \frac{1}{a}} \int_{a^{2}}^{a} \frac{r}{r^{2 \delta}} \mathrm{d} r \leq C a^{-\eta \delta+2-2 \delta} .
\end{aligned}
$$

3.1. Proof of Theorem 1.1 when $n=3$. First, consider a sequence $\left\{b_{k}\right\}_{k=1}^{\infty}$ defined by

$$
b_{k}=\frac{1}{2}\left(1+\frac{1}{k+1}\right)
$$

By applying algorithm in Section 2.4 we construct a Cantor set

$$
C_{B}:=C\left[\left\{b_{k}\right\}_{k=1}^{\infty}\right]
$$

in $[-1,1]^{3}$. Then, by $(2.2)$ we get

$$
\mathcal{L}^{3}\left(C_{B}\right)=\lim _{k \rightarrow \infty} 2^{3 k}\left(2 b_{k} 2^{-k}\right)^{3}=1
$$

Suppose that

$$
Q_{\boldsymbol{v}(k)}^{\prime}=Q\left(z_{\boldsymbol{v}(k)}, 2^{-k} b_{k-1}\right) \text { and } Q_{\boldsymbol{v}(k)}=Q\left(z_{\boldsymbol{v}(k)}, 2^{-k} b_{k}\right), \quad \boldsymbol{v}(k) \in \mathbb{V}^{k},
$$

are the corresponding cubes in the construction of the Cantor set $C_{B}$.

We will now define our homeomorphism $f$. For this, set

$$
a_{k}=\frac{1}{4} \min \left\{2^{-k^{4}},\left(\frac{1}{2}\left(b_{k-1}-b_{k}\right)\right)^{\frac{1}{\eta}}, \delta\right\} .
$$

Given $\delta<1$ we find $\eta>0$ such that

$$
-\eta \delta+2-2 \delta>0 \text {. }
$$

For every $k \in \mathbb{N}$ we consider homeomorphism as in Lemma 3.1 applied to $a=a_{k}$ scaled in each direction (both in domain and in target) by a factor of $\frac{1}{4} 2^{-k}$. We put a translated copy of such a homeomorphism in every

$$
Q_{\boldsymbol{v}}^{\prime} \backslash Q_{\boldsymbol{v}}, \boldsymbol{v} \in \mathbb{V}^{k}
$$

As $2 a_{k}^{\eta}<b_{k-1}-b_{k}$ there is enough room there (the volume of the anuloid is roughly $\left.2^{-k-2} \times 2^{-k-2} \times\left(2^{-k-2} 2\left(a_{k}\right)^{\eta}\right)\right)$. In this way we obtain $2^{3 k}$ copies of this homeomorphism for each $k \in \mathbb{N}$ and the supports of these maps are clearly disjoint. These 
homeomorphisms equal to identity on the boundaries and hence we can extend them by identity everywhere else and we obtain a homeomorphism of $(-1,1)^{3}$ onto $(-1,1)^{3}$.

We have $2^{3 k}$ cubes in the construction and by scaling by $2^{-k-2}$ in all three directions we get by Lemma 3.1 that

$$
\int_{(-1,1)^{3}}|D f|^{2} \leq C \sum_{k=1}^{\infty} 8^{k}\left(2^{-k-2}\right)^{3}\left(a_{k}^{2 \eta} \log \frac{1}{a_{k}}+C \frac{1}{\log ^{\frac{1}{2}} \frac{1}{a_{k}}}\right)<\infty
$$

as $a_{k} \leq \frac{1}{4} 2^{-k^{4}}$. Analogously we use $-\eta \delta+2-2 \delta>0$ to show that

$$
\int_{(-1,1)^{3}} K_{I}^{\delta} \leq C \sum_{k=1}^{\infty} 8^{k}\left(2^{-k-2}\right)^{3}\left(a_{k}^{2 \eta} \log \frac{1}{a_{k}}+C a_{k}^{-\eta \delta+2-2 \delta}\right)<\infty .
$$

It remains to show that $f$ is not differentiable at points of $C_{A}=C_{a} \times C_{a} \times C_{a}$. For every $x \in C_{A}$ and every $k$ we can find $\boldsymbol{v} \in \mathbb{V}^{k}$ such that $x \in Q_{\boldsymbol{v}}$. In $Q_{\boldsymbol{v}}^{\prime} \backslash Q_{\boldsymbol{v}}$ we have a translated and scaled copy of a homeomorphism as in Lemma 3.1 and hence the distance of $x$ and its support is less than $10 \cdot 2^{-k}$. For $p$ on the boundary of the corresponding anuloid we have $f(p)=p$ and as $f(x)=x$ we can see that if there is a classical derivative $D f(x)$ it must be equal to identity matrix $D f(x)=I$ as this happens on all scales $k$. On the other hand in the central circle of the anuloid our function is rotated and $|f(p)-p|=\frac{1}{2} 2^{-k}$ there. This happens on all scales $k$ and it follows that $D f(x)$ cannot be $I$ and hence $f$ is not differentiable at $x$.

3.2. Proof of the Theorem 1.1 when $n \geq 4$. The construction in the case $n \geq 4$ is very similar and therefore we only briefly outline this. We work with anuloids like

$$
R:=\left\{x \in \mathbb{R}^{n}:\left(\sqrt{x_{2}^{2}+\ldots+x_{n}^{2}}-1\right)^{2}+x_{1}^{2} \leq\left(2 a^{\eta}\right)^{2}\right\}
$$

and again $S$ has radius $a^{\eta}$ and $T$ has radius $a$. We use spherical $(n-1)$-dimensional coordinates inside the annulus (variables $r, \alpha_{1}, \ldots, \alpha_{n-2}$ ) and angle $\beta$ around the annulus as before.

We set

$$
\begin{aligned}
& f=\left[\rho(r), \alpha_{1}, \ldots, \alpha_{n-2}, \beta\right] \text { for }\left[r, \alpha_{1}, \ldots, \alpha_{n-2}, \beta\right] \in R \backslash T \text { and } \\
& f=\left[g(r), \alpha_{1}, \ldots, \alpha_{n-2}, h(r)+\beta\right] \text { for }\left[r, \alpha_{1}, \ldots, \alpha_{n-2}, \beta\right] \in T .
\end{aligned}
$$

We only sketch the estimates of the most important terms. In $T$ we have

$$
D f=\left(\begin{array}{ccccc}
g^{\prime}(r) & 0 & \ldots & 0 & h^{\prime}(r) \\
0 & \frac{g(r)}{r} & \ldots & 0 & 0 \\
\vdots & \vdots & \ddots & \vdots & \vdots \\
0 & 0 & \ldots & \frac{g(r)}{r} & 0 \\
0 & 0 & \ldots & 0 & M
\end{array}\right) \quad \text { for }\left[r, \alpha_{1}, \ldots, \alpha_{n-2}, \beta\right] \in T
$$

and hence $\left|D^{\#} f\right| \sim\left|h^{\prime}(r)\right|\left(\frac{g(r)}{r}\right)^{n-2}$ and $J_{f} \sim\left|g^{\prime}(r)\right|\left(\frac{g(r)}{r}\right)^{n-2}$ there. The important part of the derivative (where $h(t)=\log ^{\frac{1}{4}} \frac{1}{t}-\log ^{\frac{1}{4}} \frac{1}{a}$ ) can be estimated using $(n-1)$ dimensional spherical coordinates as

$$
\int|D f|^{n-1} \leq C \int_{a^{2}}^{a} \frac{1}{r^{n-1} \log ^{\frac{3}{4}(n-1)} \frac{1}{r}} r^{n-2} \mathrm{~d} r<\infty .
$$


On this part we also estimate

$$
\begin{aligned}
& \int K_{I}^{\delta} \leq C \int_{a^{2}}^{a}\left(\frac{\left|h^{\prime}(r)\right|}{a^{(n-1) \eta}}\left(\frac{g(r)}{r}\right)^{n-2}\right)^{\delta} r^{n-2} \mathrm{~d} r \\
& \leq C \int_{a^{2}}^{a}\left(\frac{1}{a^{(n-1) \eta} r \log ^{\frac{3}{4}} \frac{1}{r}} \frac{a^{(n-2) \eta}}{r^{n-2}}\right)^{\delta} r^{n-2} \mathrm{~d} r \leq C a^{-\eta \delta+n-1-(n-1) \delta} .
\end{aligned}
$$

Given $\delta<1$ we can choose $\eta>0$ such that $-\eta \delta+n-1-(n-1) \delta>0$. Other integrals can be estimated analogously as before.

Similarly we construct a Cantor type set of positive measure in $\mathbb{R}^{n}$ and in each of the $2^{k n}$ sets $Q_{\boldsymbol{v}}^{\prime} \backslash Q_{\boldsymbol{v}}, \boldsymbol{v} \in \mathbb{V}^{k}$, we put a translated and scaled (by factor $2^{-k}$ in $n$ directions) copy of the homeomorphism as above. Analogously to the computation in (3.3) we obtain using $-\eta \delta+n-1-(n-1) \delta>0$ and (3.4) our conclusion.

\section{Proof of Theorem 1.3}

4.1. Construction of the mapping. Let $\varepsilon>0$ and suppose that $n \geq 2$. We will define a homeomorphism $f:(-1,1)^{n} \rightarrow \mathbb{R}^{n}$ of finite distortion as follows:

(1) Define the sequences $\left\{a_{k}\right\}_{k=0}^{\infty}$ and $\left\{b_{k}\right\}_{k=0}^{\infty}$ by setting

$$
a_{k}=\frac{1}{(k+1)^{\alpha}} \quad \text { and } \quad b_{k}=\frac{1}{2}\left(1+\frac{1}{\log (e+k)}\right),
$$

where $\alpha>0$. It is easy to see that for every $k \geq 1$ we have

$$
a_{k-1}-a_{k} \sim \frac{1}{k^{\alpha+1}} \quad \text { and } \quad b_{k-1}-b_{k} \sim \frac{1}{k \log ^{2}(e+k)} .
$$

(2) Denote by

$$
C_{A}:=C\left[\left(a_{k}\right)_{k=0}^{\infty}\right] \quad \text { and } \quad C_{B}:=C\left[\left(b_{k}\right)_{k=0}^{\infty}\right]
$$

the Cantor sets in $\mathbb{R}^{n-1}$ given by the sequences $\left\{a_{k}\right\}_{k=0}^{\infty}$ and $\left\{b_{k}\right\}_{k=0}^{\infty}$ above. For each index $\boldsymbol{v}(k) \in \mathbb{V}^{k}, k \in \mathbb{N}$, we denote by

$$
\mathbb{A}_{\boldsymbol{v}(k)}:=Q_{\boldsymbol{v}(k)}^{\prime} \backslash \operatorname{int}\left(Q_{\boldsymbol{v}(k)}\right) \quad \text { and } \quad \mathbb{B}_{\boldsymbol{v}(k)}:=\tilde{Q}_{\boldsymbol{v}(k)}^{\prime} \backslash \operatorname{int}\left(\tilde{Q}_{\boldsymbol{v}(k)}\right)
$$

the corresponding $(n-1)$-dimensional cubical annuli in the construction of the Cantor sets $C_{A}$ and $C_{B}$. Then for $k \geq 1$ we have

$$
\begin{aligned}
& \mathcal{L}^{n}\left(\mathbb{A}_{\boldsymbol{v}(k)} \times(-1,1)\right)=2^{-k(n-1)+1}\left(a_{k-1}^{n-1}-a_{k}^{n-1}\right) \\
& =2^{-k(n-1)+1}\left(\frac{1}{(k-1)^{\alpha(n-1)}}-\frac{1}{k^{\alpha(n-1)}}\right) \sim \frac{2^{-k(n-1)}}{k^{\alpha(n-1)+1}} .
\end{aligned}
$$

and similarly

$$
\mathcal{L}^{n}\left(\mathbb{B}_{\boldsymbol{v}(k)} \times(-1,1)\right)=2^{-k(n-1)+1}\left(b_{k-1}^{n-1}-b_{k}^{n-1}\right) \sim \frac{2^{-k(n-1)}}{k \log ^{2}(e+k)} .
$$

(3) Suppose that $\Phi:(-1,1)^{n-1} \rightarrow(-1,1)^{n-1}$ is the transformation, introduced in Section 2.7, taking the Cantor set $C_{A}$ onto $C_{B}$. Define a homeomorphism $H:(-1,1)^{n} \rightarrow(-1,1)^{n}$ by

$$
H\left(x_{1}, \ldots, x_{n}\right)=\left(\Phi\left(x_{1}, \ldots, x_{n-1}\right), x_{n}\right) .
$$


Then, up to a permutation of $(n-1)$ first coordinate axes, for every point $x \in \bigcup_{\boldsymbol{v} \in \mathbb{V}^{k}} \mathbb{A}_{\boldsymbol{v}(k)}$ we have

$$
D H(x)=\left(\begin{array}{ccccccc}
C & 0 & 0 & \cdots & 0 & 0 & 0 \\
(C-D)\left(x_{2}-z_{2}\right) & D & 0 & \cdots & \vdots & \vdots & 0 \\
(C-D)\left(x_{3}-z_{3}\right) & 0 & D & \cdots & 0 & 0 & 0 \\
\vdots & \vdots & \vdots & \ddots & \vdots & \vdots & 0 \\
(C-D)\left(x_{n-2}-z_{n-2}\right) & 0 & 0 & \cdots & D & 0 & 0 \\
(C-D)\left(x_{n-1}-z_{n-1}\right) & 0 & 0 & \cdots & 0 & D & 0 \\
0 & 0 & 0 & \cdots & 0 & 0 & 1
\end{array}\right)
$$

where

$$
C=\frac{b_{k-1}-b_{k}}{a_{k-1}-a_{k}} \quad \text { and } \quad D=\frac{(1-t) b_{k}+t b_{k-1}}{(1-t) a_{k}+t a_{k-1}},
$$

for some $t \in[0,1]$ and $z$ is the corresponding center (see Section 2.7). Especially

$$
\begin{aligned}
& |D H(x)| \sim \max \left\{\frac{b_{k}}{a_{k}}, \frac{b_{k-1}-b_{k}}{a_{k-1}-a_{k}}, 1\right\} \sim k^{\alpha}, \\
& \left|D^{\sharp} H(x)\right| \sim\left(\frac{b_{k}}{a_{k}}\right)^{n-2}\left(\frac{b_{k-1}-b_{k}}{a_{k-1}-a_{k}}\right) \sim \frac{k^{\alpha(n-1)}}{\log ^{2}(e+k)}, \\
& J_{H}(x) \sim\left(\frac{b_{k}}{a_{k}}\right)^{n-2}\left(\frac{b_{k-1}-b_{k}}{a_{k-1}-a_{k}}\right) \sim \frac{k^{\alpha(n-1)}}{\log ^{2}(e+k)} .
\end{aligned}
$$

(4) Define a sequence $\left\{d_{k}\right\}_{k=1}^{\infty}$ of real numbers by

$$
d_{k}=\frac{1}{\sqrt{k \log (e+k)}},
$$

and suppose that $\mathcal{S}:(-1,1)^{n} \rightarrow \mathbb{R}^{n}$ is the oscillating snowflake mapping on the Cantor set $C_{B}$ generated by the sequence $\left\{d_{k}\right\}_{k=1}^{\infty}$ (see Section 2.9). Then for every point $x \in \bigcup_{\boldsymbol{v} \in \mathbb{V}^{k}} \mathbb{A}_{\boldsymbol{v}(k)}$ we have $H(x) \in \bigcup_{\boldsymbol{v} \in \mathbb{V}^{k}} \mathbb{B}_{\boldsymbol{v}(k)}$ and thus

$$
D \mathcal{S}(H(x))=\left(\begin{array}{ccccc}
1 & 0 & \cdots & 0 & 0 \\
0 & 1 & \cdots & 0 & 0 \\
\vdots & \vdots & \ddots & \vdots & \vdots \\
0 & 0 & \cdots & 1 & 0 \\
D_{k}^{1} & D_{k}^{2} & \cdots & D_{k}^{n-1} & 1
\end{array}\right)
$$

where $D_{k}^{j}:=\sum_{l=1}^{k} d_{l} \delta_{Q_{\left(\boldsymbol{v}_{1}, \ldots, \boldsymbol{v}_{l}\right)}^{j}}(\hat{x}) \delta\left(\boldsymbol{v}_{l}\right)$ (see Section 2.9), and therefore

$$
\begin{aligned}
& |D \mathcal{S}(H(x))|=\max _{1 \leq j \leq n-1}\left|D_{k}^{j}\right|, \quad\left|D^{\sharp} \mathcal{S}(H(x))\right|=\max \left\{1, \max _{1 \leq j \leq n-1}\left|D_{k}^{j}\right|\right\}, \\
& J_{\mathcal{S}}(H(x))=1 .
\end{aligned}
$$

Recall that the distribution of $\delta_{Q_{\left(\boldsymbol{v}_{1}, \ldots, \boldsymbol{v}_{l}\right)}^{j}}^{j}(\hat{x}) \delta\left(\boldsymbol{v}_{l}\right)$ is given by the $R B(q)$-distribution (see end of Section 2.10). By applying Khintchine's inequality Lemma 2.1 for 
a given $p>0$, we get

$$
\begin{aligned}
\mathbb{E}\left[\left(\sum_{j=1}^{n-1}\left|D_{k}^{j}\right|\right)^{p}\right] & \leq C(n, p) \sum_{j=1}^{n-1} \mathbb{E}\left[\left|D_{k}^{j}\right|^{p}\right] \lesssim \sum_{j=1}^{n-1}\left(\sum_{l=1}^{k} d_{l}^{2}\right)^{\frac{p}{2}} \\
& \lesssim \sum_{j=1}^{n-1}\left(\sum_{l=1}^{k} \frac{1}{k \log (e+k)}\right)^{\frac{p}{2}} \sim(\log \log (e+k))^{\frac{p}{2}} .
\end{aligned}
$$

(5) Define $f:(-1,1)^{n} \rightarrow \mathbb{R}^{n}$ as

$$
f(x)=(\mathcal{S} \circ H)(x) .
$$

Then, for almost every point $x \in \bigcup_{\boldsymbol{v} \in \mathbb{V}^{k}} \mathbb{A}_{\boldsymbol{v}(k)}$ we have using (4.3) that

$$
\begin{aligned}
& |D f(x)|=|D S(H(x)) D H(x)| \lesssim k^{\alpha}\left(\sum_{j=1}^{n-1}\left|D_{k}^{j}\right|\right), \\
& J_{f}(x)=J_{\mathcal{S}}(H(x)) J_{H}(x) \sim \frac{k^{\alpha(n-1)}}{\log ^{2}(e+k)}, \\
& \left|D^{\sharp} f(x)\right|=\left|D^{\sharp} \mathcal{S}(H(x)) D^{\sharp} H(x)\right| \lesssim \frac{k^{\alpha(n-1)}}{\log ^{2}(e+k)}\left(\sum_{j=1}^{n-1}\left|D_{k}^{j}\right|\right) .
\end{aligned}
$$

Especially

$$
K_{I}(x)=\frac{\left|D^{\sharp} f(x)\right|^{n}}{J_{f}(x)^{n-1}} \lesssim \frac{k^{\alpha(n-1)}}{\log ^{2}(e+k)}\left(\sum_{j=1}^{n-1}\left|D_{k}^{j}\right|\right)^{n} .
$$

4.2. Properties of the mapping. Next we will show that $f$ is a homeomorphism for which Lusin's condition $(N)$ fails on hyperplanes, $f \in W^{1, n-1-\varepsilon}\left((-1,1)^{n}, \mathbb{R}^{n}\right)$, $K_{I} \in L^{1}\left((-1,1)^{n}\right)$ and $f$ maps hyperplanes, perpendicular to $n$-th coordinate axis, to sets of infinite $(n-1)$-dimensional Hausdorff measure:

(1) It is clear that $f$ is a homeomorphism as a composed mapping of two homeomorphisms. Moreover, it is easy to see that $f$ cannot satisfy Lusin's condition $(N)$ on any hyperplane $H_{t}=\left\{x \in(-1,1)^{n}: x_{n}=t\right\}, t \in(-1,1)$, with respect to $(n-1)$-dimensional Hausdorff measure. We know that the $\mathcal{H}^{n-1}$ measure of the $(n-1)$-dimensional sets

$$
C_{B}^{t}=\left\{x \in(-1,1)^{n}:\left(x_{1}, \ldots, x_{n-1}\right) \in C_{B} \text { and } x_{n}=t\right\}
$$

is positive. Since $\mathcal{S}$ may only increase $\mathcal{H}^{n-1}$, we have for every set

$$
C_{A}^{t}=\left\{x \in(-1,1)^{n}:\left(x_{1}, \ldots, x_{n-1}\right) \in C_{A} \text { and } x_{n}=t\right\}
$$

that $\mathcal{H}^{n-1}\left(C_{A}^{t}\right)=0$ and $\mathcal{H}^{n-1}\left(f\left(C_{A}^{t}\right)\right)=\mathcal{H}^{n-1}\left(\mathcal{S}\left(C_{B}^{t}\right)\right)>0$. 
(2) Let us denote $U_{k}:=\cup_{\boldsymbol{v}(k) \in \mathbb{V}^{k}} \mathbb{A}_{\boldsymbol{v}(k)} \times(-1,1)$. By applying (4.2), (4.6) and (4.5) with exponent $p=n-1-\varepsilon$ we get

$$
\begin{aligned}
\int_{(-1,1)^{n}}|D f|^{n-1-\varepsilon} & \sim \sum_{k=1}^{\infty} \mathcal{L}^{n}\left(U_{k}\right) \mathbb{E}\left[\left\{|D f(x)|^{n-1-\varepsilon}: x \in U_{k}\right\}\right] \\
& \lesssim \sum_{k=1}^{\infty} \frac{2^{-k(n-1)}}{k^{\alpha(n-1)+1}} 2^{k(n-1)} \mathbb{E}\left[k^{\alpha(n-1-\varepsilon)}\left(\sum_{j=1}^{n-1}\left|D_{k}^{j}\right|\right)^{n-1-\varepsilon}\right] \\
& \lesssim \sum_{k=1}^{\infty} \frac{\mathbb{E}\left[\sum_{j=1}^{n-1}\left|D_{k}^{j}\right|^{n-1-\varepsilon}\right]}{k^{1+\alpha \varepsilon}} \sim \sum_{k=1}^{\infty} \frac{(\log \log (e+k))^{\frac{n-1-\varepsilon}{2}}}{k^{1+\alpha \varepsilon}}<\infty
\end{aligned}
$$

which implies that $f \in W^{1, n-1-\varepsilon}\left((-1,1)^{n}, \mathbb{R}^{n}\right)$.

(3) Similarly, by applying (4.2), (4.5) with exponent $p=n$ and (4.7) we get

$$
\begin{aligned}
\int_{(-1,1)^{n}} K_{I} & =\sum_{k=1}^{\infty} \mathcal{L}^{n}\left(U_{k}\right) \mathbb{E}\left[\left\{K_{I}(x): x \in U_{k}\right\}\right] \\
& \lesssim \sum_{k=1}^{\infty} \frac{2^{-k(n-1)}}{k^{\alpha(n-1)+1}} 2^{k(n-1)} \mathbb{E}\left[\frac{k^{\alpha(n-1)}}{\log ^{2}(e+k)}\left(\sum_{j=1}^{n-1}\left|D_{k}^{j}\right|\right)^{n}\right] \\
& \lesssim \sum_{k=1}^{\infty} \frac{\mathbb{E}\left[\sum_{j=1}^{n-1}\left|D_{k}^{j}\right|^{n}\right]}{k \log ^{2}(e+k)} \sim \sum_{k=1}^{\infty} \frac{(\log \log (e+k))^{\frac{n}{2}}}{k \log ^{2}(e+k)}<\infty
\end{aligned}
$$

which implies that $K_{I} \in L^{1}\left((-1,1)^{n}\right)$.

(4) Suppose that $t \in(-1,1)$. Then for the image of the $(n-1)$-dimensional hyperplane $H_{t}=\left\{(\hat{x}, t): \hat{x} \in\left(-\frac{8}{10}, \frac{8}{10}\right)^{n-1}\right\}$ we have that

$$
\begin{aligned}
& \mathcal{H}^{n-1}\left(f\left(H_{t}\right)\right)=\mathcal{H}^{n-1}\left(\mathcal{S}\left(H_{t}\right)\right)=\lim _{N \rightarrow \infty} \mathcal{H}^{n-1}\left(\mathcal{S}_{N}\left(H_{t}\right)\right) \gtrsim \lim _{N \rightarrow \infty} \sum_{\boldsymbol{v} \in \mathbb{V} N} \mathcal{H}^{n-1}\left(\mathcal{S}_{N}\left(Q_{\boldsymbol{v}(k)}\right)\right) \\
& =\lim _{N \rightarrow \infty} \mathcal{H}^{n-1}\left(\bigcup_{\boldsymbol{v} \in \mathbb{V}^{N}} Q_{\boldsymbol{v}(k)}\right) \mathbb{E}\left(\left\{\left|D \mathcal{S}_{N}(y)\right|: y \in \bigcup_{\boldsymbol{v} \in \mathbb{V} N} Q_{\boldsymbol{v}(k)} \times\{t\}\right\}\right) \\
& \sim \lim _{N \rightarrow \infty} 2^{N(n-1)}\left(2 b_{k} 2^{-N}\right)^{n-2} b_{k} 2^{-k} \mathbb{E}\left(\left|D \mathcal{S}_{N}\right|\right) \sim \lim _{N \rightarrow \infty} \mathbb{E}\left(\left|D \mathcal{S}_{N}\right|\right)
\end{aligned}
$$

and it remains to show that this expected value of derivative is infinite. Using (4.4) it is enough to show that for every $j \in\{1, n-1\}$ we have using Khintchine inequality Lemma 2.1 for $p=1$ similarly to (4.5)

$$
\mathbb{E}\left(\left|D_{N}^{j}\right|\right) \gtrsim\left(\sum_{l=1}^{N} d_{l}^{2}\right)^{\frac{1}{2}} \sim(\log \log (e+N))^{\frac{1}{2}} \stackrel{N \rightarrow \infty}{\rightarrow} \infty .
$$

Thus $\mathcal{H}^{n-1}\left(f\left(H_{t}\right)\right)=\infty$.

4.3. Failure of the $K_{I^{-}}$-inequality for $f$. In this section we will show that $K_{I^{-}}$ inequality (1.1) fails for $f$ which will end the proof of Theorem 1.3. The proof of

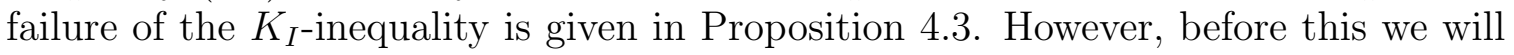
give two technical lemmata which we then apply in the proof of Proposition 4.3. 
Lemma 4.1. Let $A \subset \mathbb{R}^{n-1}$ be an open and bounded set. Suppose that $u_{0}: A \rightarrow \mathbb{R}$ is a linear affine function and for each $h \geq 0$ define $u_{h}: A \rightarrow \mathbb{R}$ as

$$
u_{h}(x)=u_{0}(x)+h .
$$

Suppose that $\mathcal{G}_{u_{h}}^{A}=\left\{\left(x, u_{h}(x)\right): x \in A\right\}$ is the graph of $u_{h}$ on $A$, and let $\mathbf{n}$ be the unit normal vector of the surface $\mathcal{G}_{u_{0}}$. Denote by

$$
S_{h}:=\left\{z \in \mathcal{G}_{u_{0}}^{A}: z+\mathbf{n} t \in \mathcal{G}_{u_{h}}^{A} \text { for some } t \in \mathbb{R}\right\}
$$

the shadow of the set $\mathcal{G}_{u_{h}}^{A}$ on $\mathcal{G}_{u_{0}}^{A}$. Then for a given $\varepsilon>0$ there exist $h_{\varepsilon}>0$ such that

$$
\mathcal{H}^{n-1}\left(S_{h}\right) \geq(1-\varepsilon) \mathcal{H}^{n-1}\left(\mathcal{G}_{u_{0}}^{A}\right)
$$

for all $0 \leq h<h_{\varepsilon}$.

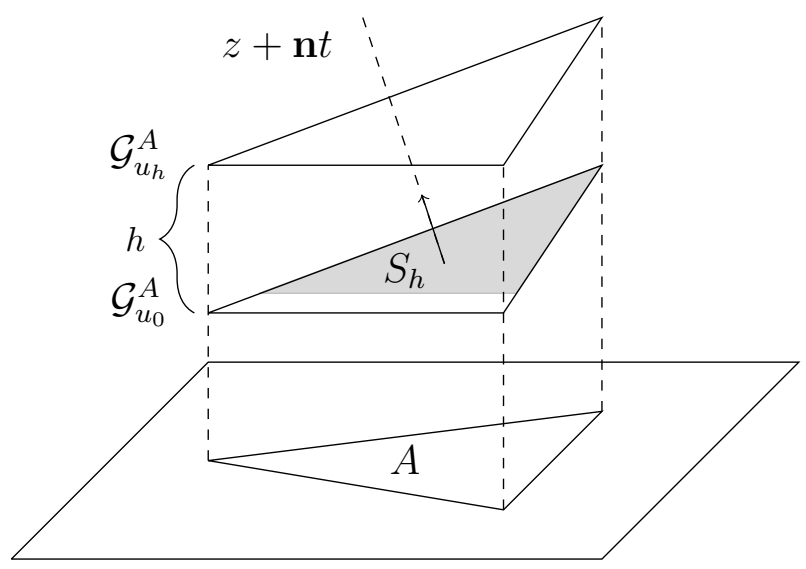

Fig. 7. The shadow $S_{h}$ of the graph $\mathcal{G}_{u_{h}}^{A}$ on $\mathcal{G}_{u_{0}}^{A}$.

Lemma 4.1 follows easily from the fact $S_{h} \nearrow S_{0}$, and we leave the proof for the reader. In the next lemma we show that if we have some linear function and a small slab between a small shift of this function, then it actually almost minimizes some capacity among all continuous deformations of this slab.

Lemma 4.2. Let $A \subset \mathbb{R}^{n-1}$ be an open set and let $D \subset \subset A$ be an open and convex subset of $A$. Suppose that $u: A \rightarrow \mathbb{R}$ is a linear affine function, and let $f: A \rightarrow \mathbb{R}$ be an arbitrary continuous function such that

$$
f(x)=u(x) \quad \text { for all } x \in A \backslash D .
$$

For $h>0$ and for a given continuous function $g: A \rightarrow \mathbb{R}$ define a number

$$
C_{h}(g):=\inf \left\{\int_{D \times \mathbb{R}}|\nabla v(x)|^{n} \mathrm{~d} x: v=0 \text { on } \mathcal{G}_{g}^{D} \text { and } v \geq 1 \text { on } \mathcal{G}_{g+h}^{D}\right\},
$$

Then there exists $h_{0}>0$ such that

$$
C_{h}(u) \leq 2^{n} C_{h}(f),
$$

for all $0<h<h_{0}$. 
Proof. Let $0<\varepsilon<1 / 2$, and suppose that $S_{h}$ is the shadow of the set $\mathcal{G}_{u+h}^{D}$ on $\mathcal{G}_{u}^{D}$. Due to Lemma 4.1 we may assume that there exist $h_{\varepsilon}>0$ such that

$$
\mathcal{H}^{n-1}\left(\mathcal{G}_{u}^{D}\right)<\frac{\mathcal{H}^{n-1}\left(S_{h}\right)}{1-\varepsilon}
$$

for all $0<h<h_{\varepsilon}$. Let us denote $l:=\operatorname{dist}\left(\mathcal{G}_{u}^{D}, \mathcal{G}_{u+h}^{D}\right)$. By applying Fubini's theorem and (4.9) we get

$$
h \mathcal{H}^{n-1}(D)=\int_{D}(u+h)-\int_{D} u=l \mathcal{H}^{n-1}\left(\mathcal{G}_{u}^{D}\right)<\frac{l \mathcal{H}^{n-1}\left(S_{h}\right)}{1-\varepsilon}
$$

for all $0<h<h_{\varepsilon}$.

Let $\mathbf{n}$ be the unit normal vector of $\mathcal{G}_{u}^{D}$ pointing in the direction of the set $\mathcal{G}_{u+h}^{D}$. Suppose that $v: D \times \mathbb{R} \rightarrow[0,1]$ is an admissible test function for $C_{h}(u)$ such that

(i) $v(x+t \mathbf{n})=\frac{t}{l}$ for all points $x \in \mathcal{G}_{u}^{D}$ and for all $t \in(0, l)$,

(ii) $v(x)=0$ for all points $x \in D \times \mathbb{R}$ which are bellow the graph $\mathcal{G}_{u}^{D}$, and

(iii) $v(x)=1$ for all points $x \in D \times \mathbb{R}$ which are above the graph $\mathcal{G}_{u+h}^{D}$.

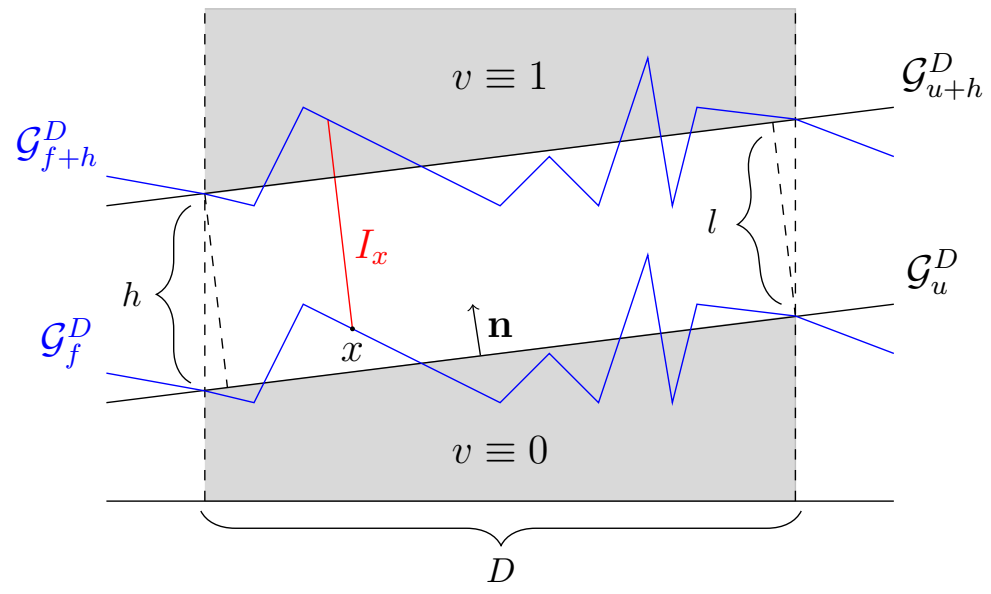

Fig. 8. Setting in the Lemma 4.2.

Then it follows from (4.9) that for $0<\varepsilon<1 / 2$ we have using $|\nabla v|=\frac{1}{l}$

$$
C_{h}(u) \leq \int_{D \times \mathbb{R}}|\nabla v|^{n}=\int_{\mathcal{G}_{u}^{D}} \frac{l}{l^{n}}=\frac{\mathcal{H}^{n-1}\left(\mathcal{G}_{u}^{D}\right)}{l^{n-1}}<\frac{2 \mathcal{H}^{n-1}\left(S_{h}(u)\right)}{l^{n-1}}
$$

for all $0<h<h_{\varepsilon}$.

Let $f: A \rightarrow \mathbb{R}$ be a continuous function as in the statement of lemma. Then we may define a mapping $F: A \times \mathbb{R} \rightarrow \mathbb{R}^{n}$ by setting

$$
F\left(x_{1}, \ldots, x_{n}\right)=\left(x_{1}, \ldots, x_{n-1}, x_{n}+f\left(x_{1}, \ldots, x_{n-1}\right)\right) .
$$

If we apply (4.10), we get

$$
\mathcal{H}^{n}(F(D \times(0, h)))=\int_{D}(f+h)-\int_{D} f=h \mathcal{H}^{n-1}(D)<\frac{l \mathcal{H}^{n-1}\left(S_{h}\right)}{1-\varepsilon},
$$

for all $0<h<h_{\varepsilon}$. For each point $x \in \mathcal{G}_{u}^{D}$ let us define the 1-dimensional set $I_{x}$ as

$$
I_{x}=\{z \in F(D \times(0, h)): z=x+t \mathbf{n} \text { for some } t \in \mathbb{R}\} .
$$


Then, if $w$ is an admissible test function for $C_{h}(f)$, we have

$$
\int_{I_{x}}|\nabla w| \geq 1
$$

for every $x \in S_{h}$. By integrating both sides over the set $S_{h}$, using Hölder's inequality, Fubini's theorem and (4.12) we have

$$
\begin{aligned}
\mathcal{H}^{n-1}\left(S_{h}\right) \leq \int_{S_{h}} \int_{I_{x}}|\nabla w| & \leq\left(\mathcal{H}^{n}(F(D \times(0, h)))\right)^{\frac{n-1}{n}}\left(\int_{D \times \mathbb{R}}|\nabla w|^{n}\right)^{\frac{1}{n}} \\
& <\left(\frac{l \mathcal{H}^{n-1}\left(S_{h}\right)}{1-\varepsilon}\right)^{\frac{n-1}{n}}\left(\int_{D \times \mathbb{R}}|\nabla w|^{n}\right)^{\frac{1}{n}} .
\end{aligned}
$$

Thus, by assuming $0<\varepsilon<1 / 2$ we get $\frac{\mathcal{H}^{n-1}\left(S_{h}\right)}{2^{n-1} l^{n-1}} \leq \int_{D \times \mathbb{R}}|\nabla w|^{n}$, and if we take infimum over all admissible test functions $w$, we conclude that

$$
\frac{\mathcal{H}^{n-1}\left(S_{h}\right)}{2^{n-1} l^{n-1}} \leq C_{h}(f),
$$

for all $0<h<h_{\varepsilon}$. Claim follows now from (4.11) and (4.13).

Proposition 4.3. Let $\delta:=\frac{1}{10}$ and let $f$ be the mapping given in Section 4.1. For a given $h>0$ define a condenser $\left(E_{h}, \bar{E}_{0}\right)$ by setting

$$
\begin{aligned}
& E_{0}:=(-1+2 \delta, 1-2 \delta)^{n-1} \times(-1+2 \delta, 0), \text { and } \\
& E_{h}:=(-1+\delta, 1-\delta)^{n-1} \times(-1+\delta, h) .
\end{aligned}
$$

Then

$$
\lim _{h \rightarrow 0} \frac{\operatorname{cap}\left(f\left(E_{h}\right), f\left(\bar{E}_{0}\right)\right)}{\operatorname{cap}_{K_{I}}\left(E_{h}, \bar{E}_{0}\right)}=\infty .
$$

Proof. Fix $M \geq 1$. For $t \in \mathbb{R}$ denote $\hat{E}_{t}=(-1+2 \delta, 1-2 \delta)^{n-1} \times\{t\}$, and whenever $t \neq s$ define

$$
\widehat{\operatorname{cap}}\left(f\left(\hat{E}_{t}\right), f\left(\hat{E}_{s}\right)\right)=\inf \int_{(-1,1)^{n-1} \times \mathbb{R}}|\nabla u(x)|^{n} \mathrm{~d} x,
$$

where the infimum is taken over all functions $u \in C^{\infty}\left((-1,1)^{n-1} \times \mathbb{R}\right)$ such that $\left.u\right|_{f\left(\hat{E}_{t}\right)} \equiv 0$ and $\left.u\right|_{f\left(\hat{E}_{s}\right)} \equiv 1$.

By $\delta=\frac{1}{10}$ we know that $C_{A} \subset(-1+\delta, 1-\delta)^{n-1}$, where $C_{A}$ is the given Cantor set of zero measure in the construction of $f$. Then it is easy to see that it suffices to show that there is $h_{M}>0$ such that

$$
\frac{\widehat{\operatorname{cap}}\left(f\left(\hat{E}_{h}\right), f\left(\hat{E}_{0}\right)\right)}{\operatorname{cap}_{K_{I}}\left(E_{h}, \bar{E}_{0}\right)} \geq M,
$$

for all $0<h<h_{M}$.

To prove (4.14) suppose that $u$ is any test function which goes linearly from 1 to 0 along the line segments parallel to the $n$th coordinate axes. We know by the construction that $f=\mathcal{S} \circ H$ behaves the same way on each hyperplane and hence the 
integral of $K_{I}$ over every $(n-1)$-dimensional hyperplane $(-1,1)^{n-1} \times\{t\}, 0<t<h$, is the same (see Section 4.1). By applying Fubini's theorem we get

$$
\begin{aligned}
\operatorname{cap}_{K_{I}}\left(E_{h}, \bar{E}_{0}\right) \leq \int_{(-1,1)^{n-1} \times(0, h)}|\nabla u(x)|^{n} K_{I}(x) \mathrm{d} x \\
\quad=\int_{(-1,1)^{n-1} \times(0, h)} \frac{K_{I}(x)}{h^{n-1}} \mathrm{~d} x=\frac{1}{h^{n}} \int_{0}^{h} \int_{(-1,1)^{n-1} \times\{t\}} K_{I} \leq \frac{C_{0}}{h^{n-1}},
\end{aligned}
$$

where the finite constant $C_{0}:=C_{0}\left(n, \delta, K_{I}\right) \geq 1$ is depending only on $n, \delta$ and on the integral of $K_{I}$ over the level set $(-1,1)^{n-1} \times\{0\}$.

Next, we denote $f_{N}:=\mathcal{S}_{N} \circ H$. Then the calculation in Section 4.2 (4) shows us that there is $N_{0} \geq 1$ such that

$$
\mathcal{H}^{n-1}\left(f_{N}\left(\hat{E}_{0}\right)\right)=\mathcal{H}^{n-1}\left(\mathcal{S}_{N}\left(\hat{E}_{0}\right)\right)>2^{n+1} M C_{0}
$$

for all $N \geq N_{0}$. Let us fix $N \geq N_{0}$.

As the mapping in the construction of $S_{k}$ are piecewise affine, we may divide the sets $\hat{E}_{0}$ and $\hat{E}_{h}$ into finitely many pairwise disjoint, open, convex and maximal $(n-1)$ dimensional sets $\left\{E_{0}^{j}\right\}_{j=1}^{l}$ and $\left\{E_{h}^{j}\right\}_{j=1}^{l}$ such that:

(i) $\hat{E}_{0}=\bigcup_{j=1}^{l} \bar{E}_{0}^{j}$ and $\hat{E}_{h}=\bigcup_{j=1}^{l} \bar{E}_{h}^{j}$, where the closure is taken in $(-1,1)^{n-1} \times \mathbb{R}$ instead of $\mathbb{R}^{n}$.

(ii) $E_{h}^{j}=E_{0}^{j}+h$ for all $j=1, \ldots, l$.

(iii) Sets $E_{0}^{j}, j=1, \ldots, l$, are not depending on $h$.

(iv) The restriction of $\mathcal{S}_{N}$ on each set $E_{0}^{j}$ and $E_{h}^{j}$ can be written as

$$
\begin{aligned}
& \mathcal{S}_{N}(\hat{x})=\left(\hat{x}, L_{j}(\hat{x})\right) \quad \text { for all } \hat{x} \in E_{0}^{j} \text { and } \\
& \mathcal{S}_{N}(\hat{x})=\left(\hat{x}, L_{j}(\hat{x})+h\right) \quad \text { for all } \hat{x} \in E_{h}^{j},
\end{aligned}
$$

where $L_{j}: E_{0}^{j} \rightarrow \mathbb{R}$ is a linear affine function.

Fix $\varepsilon \in(0,1 / 2)$. Denote by $\mathbf{n}_{j}$ the unit normal vector of $\mathcal{G}_{L_{j}}^{E_{0}^{j}}$ and $S_{h}^{j}$ the shadow of the graph $\mathcal{G}_{L+h}^{E_{0}^{j}}$ on $\mathcal{G}_{L}^{E_{0}^{j}}$ (see Lemma 4.1). Then by applying Lemma 4.1 and (4.16) we may find $h_{\varepsilon}>0$ such that

$$
\begin{gathered}
\sum_{j=1}^{l} \mathcal{H}^{n-1}\left(S_{h}^{j}\right) \geq(1-\varepsilon)\left(\sum_{j=1}^{l} \mathcal{H}^{n-1}\left(\mathcal{G}_{L_{j}}^{E_{0}^{j}}\right)\right)=(1-\varepsilon)\left(\sum_{j=1}^{l} \mathcal{H}^{n-1}\left(\mathcal{S}_{N_{0}}\left(E_{0}^{j}\right)\right)\right) \\
=(1-\varepsilon) \mathcal{H}^{n-1}\left(\mathcal{S}_{N_{0}}\left(E_{0}\right)\right)>2^{n} M C_{0}
\end{gathered}
$$

for all $0<h<h_{\varepsilon}$. Fix $0<h<h_{\varepsilon}$ and for each $j=1, \ldots, l$ define

$$
\begin{array}{r}
\mathcal{I}_{j}^{h}=\left\{I_{z}: I_{z} \text { is a line segment parallel to } \mathbf{n}_{j} \text { starting at a point } z \in S_{h}^{j}\right. \\
\text { and ending to a point } \left.z+\mathbf{n}_{j} t \in \mathcal{G}_{L+h}^{E_{0}^{j}} \text { for some } t \in \mathbb{R}\right\} .
\end{array}
$$

Then, if $u$ is an admissible test function for $\widehat{\operatorname{cap}}\left(f_{N}\left(\hat{E}_{0}\right), f_{N}\left(\hat{E}_{h}\right)\right)$, we have

$$
\int_{I}|\nabla u| \geq 1 \quad \text { for all } I \in \cup_{j=1}^{l} \mathcal{I}_{j} .
$$


Especially, by integrating over each $(n-1)$-dimensional set $S_{h}^{j}$, applying Hölder's inequality and using the fact that the length of each line segment $I \in \cup_{j=1}^{l} \mathcal{I}_{j}$ is at most $h$, we get

$$
\begin{aligned}
\sum_{j=1}^{l} \mathcal{H}^{n-1}\left(S_{h}^{j}\right) & \leq \sum_{j=1}^{l} \int_{S_{h}^{j}} \int_{I_{z}}|\nabla u|=\int_{\cup_{j=1}^{l} S_{h}^{j}} \int_{I_{z}}|\nabla u| \\
& \leq\left(h \sum_{j=1}^{l} \mathcal{H}^{n-1}\left(S_{h}^{j}\right)\right)^{\frac{n-1}{n}}\left(\int|\nabla u|^{n}\right)^{\frac{1}{n}}
\end{aligned}
$$

which implies together with (4.17) that

$$
\frac{2^{n} M C_{0}}{h^{n-1}} \leq \int|\nabla u(x)|^{n} \mathrm{~d} x
$$

Thus, by taking infimum over all admissible test functions $u$ we get

$$
\widehat{\operatorname{cap}}\left(f_{N}\left(\hat{E}_{h}\right), f_{N}\left(\hat{E}_{0}\right)\right) \geq \frac{2^{n} M C_{0}}{h^{n-1}} .
$$

The first $n-1$ coordinate functions of $f$ agree with first $n-1$ coordinate functions of $f_{N}$ and the last coordinate mapping of $f$ agrees with that coordinate of piecewise linear mapping $f_{N}$ close to $\partial(-1+2 \delta, 1-2 \delta)^{n-1} \times\left(0, h_{0}\right)$. Hence we can apply Lemma 4.2 (for the last coordinate mapping) and (4.18) to derive

$$
\operatorname{cap}\left(f\left(\hat{E}_{h}\right), f\left(\hat{E}_{0}\right)\right) \geq 2^{-n-1} \operatorname{cap}\left(f_{N}\left(\hat{E}_{h}\right), f_{N}\left(\hat{E}_{0}\right)\right) \geq \frac{M C_{0}}{h^{n-1}} .
$$

Finally, by putting together estimates in (4.15) and (4.19) we get

$$
\frac{\widehat{\operatorname{cap}}\left(f\left(\hat{E}_{h}\right), f\left(\hat{E}_{0}\right)\right)}{\operatorname{cap}_{K_{I}}\left(E_{h}, E_{0}\right)} \geq M,
$$

and the claim follows.

Acknowledgement. The authors would like to thank Changyu Guo and Tomáš Roskovec for their valuable comments. They would also like to thank Petr Honzík for pointing out the authors interest to Khinchine inequality.

\section{REFERENCES}

[1] M. Csörnyei, S. Hencl and J. Malý, Homeomorphisms in the Sobolev space $W^{1, n-1}$, J. Reine Angew. Math. 644 (2010), 221-235.

[2] F. W. Gehring and O. Lehto, On the total differentiability of functions of a complex variable, Ann. Acad. Sci. Fenn. Ser. A1 272 (1959), 1-9.

[3] F. W. Gehring and J. Väisälä, The coefficients of quasiconformality of domains in space, Acta Math. 114 (1965), 1-70.

[4] S. Hencl and P. Koskela, Lectures on Mappings of finite distortion, Lecture Notes in Mathematics 2096, Springer, Cham, 2014, 176pp.

[5] S. Hencl, P. Koskela and J. Malý, Regularity of the inverse of a Sobolev homeomorphism in space, Proc. Roy. Soc. Edinburgh Sect. A 136, no. 6, (2006), 1267-1285.

[6] A. Khintchine, Über dyadische Brüche, Math. Z. 18, no. 1, (1923), 109-116.

[7] P. Koskela and J. Onninen, Mappings of finite distortion: Capacity and modulus inequalities, J. Reine Angew. Math. 599 (2006), 1-26.

[8] O. Martio, On the integrability of the derivative of a quasiregular mapping, Math. Scand. 35 (1974), 43-48. 
[9] O. Martio, A capacity inequality for quasiregular mappings, Ann. Acad. Sci. Fenn. Ser. A I 474 (1970), 1-18.

[10] O. Martio, S. Rickman and J. Väisälä, Definitions for quasiregular mappings, Ann. Acad. Sci. Fenn. Ser. A I 448 (1969), 1-40.

[11] G. Moscariello and A. Passarelli di Napoli, The regularity of the inverse of Sobolev homeomorphism with finite distortion, J. Geom. Anal. 24, no. 1, (2014), 571-594.

[12] J. Onninen, Regularity of the inverse of spatial mappings with finite distortion, Cal. Var. Partial Differential Equations 26, no. 3, (2006), 331-341.

[13] J. Onninen, Differentiability of monotone Sobolev functions, Real Anal. Exchange 26, no. 2, (2000), 761-772.

[14] S. Rickman, Quasiregular mappings, Ergebnisse der Mathematik und ihrer Grenzgebiete (3) [Results in Mathematics and Related Areas (3)], 26. Springer-Verlag, Berlin, 1993.

[15] R. Salimov, ACL and differentiability of Q-homeomorphisms, Ann. Acad. Sci. Fenn. Math. 33, no. 1, (2008), 295-301.

[16] R.R. Salimov and E.A. Sevost'yanov, ACL and differentiability of open discrete ring $(p, Q)$ mappings, Mat. Stud. 35, no. 1, (2011), 28-36.

[17] T. Tengvall, Differentiability in the Sobolev space $W^{1, n-1}$, Cal. Var. Partial Differential Equations 51, no. 1-2, (2014), 381-399.

[18] J. Väisälä, Lectures on n-dimensional quasiconformal mappings, Lecture Notes in Mathematics, Vol. 229, Springer-Verlag, Berlin, 1971.

[19] T.H. Wolff, Lectures in harmonic analysis, http://www.math.ubc.ca/ ilaba/wolff/notes_march2002.pdf, 2002.

[20] W. P. Ziemer, Extremal length and conformal capacity, Trans. Amer. Math. Soc. 126 (1967), 460-473.

[21] W. P. Ziemer, Extremal length and p-capacity, Michigan Math. J. 16 (1969), 43-51.

Department of Mathematical Analysis, Charles University, Sokolovská 83, 18600 Prague 8, Czech Republic

E-mail address: hencl@karlin.mff.cuni.cz; ville.tengvall@jyu.fi 